\title{
Piecewise log-normal approximation of size distributions for aerosol modelling
}

\author{
K. von Salzen \\ Canadian Centre for Climate Modelling and Analysis, Science and Technology Branch, Environment Canada, Victoria, \\ British Columbia, Canada
}

Received: 7 April 2005 - Published in Atmos. Chem. Phys. Discuss.: 14 June 2005

Revised: 14 December 2005 - Accepted: 21 February 2006 - Published: 27 April 2006

\begin{abstract}
An efficient and accurate method for the representation of particle size distributions in atmospheric models is proposed. The method can be applied, but is not necessarily restricted, to aerosol mass and number size distributions. A piecewise log-normal approximation of the number size distribution within sections of the particle size spectrum is used. Two of the free parameters of the log-normal approximation are obtained from the integrated number and mass concentration in each section. The remaining free parameter is prescribed. The method is efficient in a sense that only relatively few calculations are required for applications of the method in atmospheric models.

Applications of the method in simulations of particle growth by condensation and simulations with a single column model for nucleation, condensation, gravitational settling, wet deposition, and mixing are described. The results are compared to results from simulations employing singleand double-moment bin methods that are frequently used in aerosol modelling. According to these comparisons, the accuracy of the method is noticeably higher than the accuracy of the other methods.
\end{abstract}

\section{Introduction}

Models such as atmospheric general circulation models (AGCMs) and air quality models often lack the ability to predict aerosol size distributions mainly because of the large computational costs that are associated with a prognostic treatment of aerosol dynamical processes. Instead, parameterizations of aerosol chemical and physical processes are commonly based on highly idealized size distributions, for example, with constant size parameters. However, aerosol size distributions are considerably variable over a wide range

Correspondence to: K. von Salzen

(knut.vonsalzen@ec.gc.ca) of spatial and temporal scales in the atmosphere (Gilliani et al., 1995; von Salzen et al., 2000; Birmili et al., 2001). Unaccounted variability in size distributions is a primary source of uncertainty in studies of aerosol effects on clouds and climate (e.g. Feingold, 2003).

Consequently, accurate and computationally efficient numerical methods are required for the representation of aerosol size distributions in aerosol models.

Observed aerosol number size distributions can often be well approximated by a series of overlapping modes, with the size distribution for each mode represented by the lognormal distribution, i.e.,

$$
\begin{aligned}
& n\left(R_{p}\right)=\frac{\mathrm{d} N}{\mathrm{~d} \ln \left(R_{p} / R_{0}\right)} \\
& =\frac{N}{\sqrt{2 \pi} \ln \sigma} \exp \left\{-\frac{\left[\ln \left(R_{p} / R_{0}\right)-\ln \left(R_{g} / R_{0}\right)\right]^{2}}{2 \ln ^{2} \sigma}\right\},
\end{aligned}
$$

with particle radius $R_{p}$, mode mean particle radius $R_{g}$, total number of particles per unit volume (or mass) $N$, and geometric standard deviation $\sigma$ (e.g. Whitby, 1978; Seinfeld and Pandis, 1998; Remer et al., 1999). $R_{0}$ is an arbitrary reference radius (e.g. $1 \mu \mathrm{m}$ ) that is required for a dimensionally correct formulation.

The so-called modal approach in aerosol modelling is based on the idea that aerosol size distributions can be approximated using statistical moments with idealized properties (Whitby, 1981, 1997; Binkowski and Shankar, 1995; Wilck and Stratmann, 1997; Ackermann et al., 1998; Harrington and Kreidenweis, 1998; Wilson et al., 2001). Typically, a number of overlapping log-normally distributed modes (e.g. for nucleation, accumulation and coarse mode) are assumed to account for different types of aerosols and processes in the atmosphere. An important variant of the modal approach is the quadrature method of moments, QMOM (McGraw, 1997; Wright et al., 2001; Yu et al.,

Published by Copernicus GmbH on behalf of the European Geosciences Union. 
2003), which is not necessarily constrained to log-normally distributed modes.

In practice, the log-normal distribution often yields good approximations of size distributions with clearly distinct modes under ideal conditions. However, it has been recognized that transport and non-linear dynamical processes, such as aerosol activation, condensation, and chemical production can lead to substantially skewed or even discontinuous size distributions (e.g. Kerminen and Wexler, 1995; Gilliani et al., 1995; von Salzen and Schlünzen, 1999b; Zhang et al., 2002). Skewed size distributions are often related to cloud processes so that an incomplete accounting of skewness in aerosol size distributions is a substantial concern for studies of the indirect effects of aerosols on climate.

Size distributions with non-ideal features can be well approximated with the bin approach, also often called "sectional" or "discrete" approach (Gelbard and Seinfeld, 1980; Warren and Seinfeld, 1985; Gelbard, 1990; Seigneur, 1982; Jacobson, 1997; Lurmann et al., 1997; Russell and Seinfeld, 1998; Meng et al., 1998; von Salzen and Schlünzen, 1999a; Pilinis et al., 2000; Gong et al., 2003). According to the bin approach, a range of particle sizes is subdivided into a number of bins, or sections, with discrete values of the size distribution in each bin.

It can be generally distinguished between single- and multi-moment bin schemes, depending on the number of statistical moments of the size distribution that are predicted.

In its original form with size-independent sub-bin particle size distributions (e.g. Gelbard and Seinfeld, 1980), the single-moment approach is subject to significant numerical diffusion when applied to simulations of aerosol dynamical processes (e.g. Seigneur et al., 1986). However, an even more substantial disadvantage of the method is that the application of the method to the continuity equation for a single moment (e.g. particle mass) is not sufficient to ensure conservation of other moments (e.g. particle number). Other moments of the size distribution can be obtained from diagnostic relationships that are based on the predicted mass size distribution. Owing to the lack of a prognostic treatment, the diagnosed quantities can violate fundamental mathematical constraints, such as inequality relations and positive definiteness (Feller, 1971). These can be significant problems if an insufficient number of bins is used. Despite these limitations, singlemoment schemes are still widely used in aerosol models.

Numerous multi-moment schemes have been suggested as improvements of the basic single-moment bin approach. For example, Tzivion et al. (1987) used bin-integrated aerosol number and mass concentrations to obtain sub-bin aerosol number and size distributions as linear functions of particle mass. In another example, Jacobson (1997) assigned the binintegrated aerosol number and mass concentrations to a single particle size in each bin. According to this approach, parameterizations of aerosol dynamical processes are applied to the single-particle representation of the size distribution in each bin. Transfer of particles between different bins is accomplished by combining particles with different sizes to produce a new distribution for a single particle size in each bin.

Several studies compared results of the modal and bin approaches based on a limited number of simulations of aerosol dynamical processes (e.g. Seigneur et al., 1986; Zhang et al., 1999, 2002; Wilson et al., 2001; Korhonen et al., 2003). According to the studies, the modal approach performed well in terms of computationally efficiency and is able to reproduce important features of the size distributions. However, results with this approach were noticeably biased in simulations of coagulation and condensational growth in some of the studies. On the other hand, the bin approach produced acceptable results for a relatively large number of bins. Computational efficiencies were generally lower for this approach in comparison to the modal approach.

Both approaches have certain constraints that are problematic for the development of parameterizations in models.

For the bin method, the need to use a relatively large number of bins for sufficiently accurate solutions potentially leads to considerable computational costs. For example, simulations of advection and horizontal diffusion cause an overhead of about $4 \%$ in CPU time for each additional prognostic tracer in the current operational version of the Canadian Centre for Climate Modelling and Analysis (CCCma) AGCM3 (McFarlane et al., 2006). Parameterizations of aerosol dynamical processes may lead to further and disproportional increases in CPU time.

A disadvantage for application of the modal approach in general atmospheric models is the need to account for varying degrees of overlap between different modes. As already pointed out, transport and aerosol dynamical processes often lead to changes in overlap and shape of modes. Relatively complicated treatments of aerosol dynamical processes have been proposed to allow distinct modes to merge into a single mode, for example (e.g. Harrington and Kreidenweis, 1998; Whitby et al., 2002).

It is argued here that certain advantages offered by the modal approach (e.g. observed aerosols are often nearly lognormally distributed in parts of the size spectrum) and the single-moment bin approach (e.g. flexibility and simplicity) can be exploited for the development of a hybrid method with considerably improved performance.

The mathematical framework of a new method that is based on a combination of the bin and the modal approach is described in Sect. 2. An application of the method to observed size distributions and in simulations with a single column model of the atmosphere are described in Sects. 3 and 5. 


\section{Formulation of the approximation method}

\subsection{Approximation of the aerosol number distribution}

Without any loss of generality, an approximation of an aerosol number distribution may be expressed as a series of orthogonal functions, i.e.,

$n(\varphi)=\sum_{i} n_{i}(\varphi)$

for the dimensionless size parameter $\varphi \equiv \ln \left(R_{p} / R_{0}\right)$.

The following set of functions in Eq. (1) is proposed:

$$
\begin{aligned}
n_{i}(\varphi)= & n_{0, i} \exp \left[-\psi_{i}\left(\varphi-\varphi_{0, i}\right)^{2}\right] \\
& H\left(\varphi-\varphi_{i-1 / 2}\right) H\left(\varphi_{i+1 / 2}-\varphi\right),
\end{aligned}
$$

where $n_{0, i}, \psi_{i}$, and $\varphi_{0, i}$ are fitting parameters that determine the magnitude, width, respectively mean of the distribution (Sect. 2.2). $H(x)$ denotes the Heaviside step function, i.e.

$H(x)= \begin{cases}0 & \text { if } x<0 \\ \frac{1}{2} & \text { if } x=0 \\ 1 & \text { if } x>0\end{cases}$

so that $n_{i}=0$ outside each section $i$ with the size range $\varphi_{i-1 / 2} \leq \varphi \leq \varphi_{i+1 / 2}$. Log-normal distributions with varying properties between sections are used as basis functions in each section (Eq. 2). Hence, Eqs. (1) and (2) define a piecewise log-normal approximation (PLA) of the aerosol number distribution.

As will be demonstrated in the following sections, Eqs. (1) and (2) can be directly used for the approximation of observed size distributions and development of parameterizations in models.

Note that similar to the single-moment bin approach (e.g., which can be obtained for $\psi_{i}=0$ ), the approach in Eq. (2) ensures that $n(\varphi) \geq 0$ for any given value of $\varphi$. This is not necessarily the case for other potential prescriptions, e.g. a representation of the basis functions in terms of polynomials. Additionally, the PLA method presented here has the advantages that the method is exact for strictly log-normally distributed aerosol number concentrations and that it is straightforward to calculate any higher moment of the approximated size distribution. The latter is particularly important for potential applications of the method to the development of parameterizations. Finally, approximated size distributions generally converge to the exact solution for a sufficiently large number of sections for appropriate choices of the fitting parameters.

\subsection{Determination of the fitting parameters}

The fitting parameters $n_{0, i}, \psi_{i}$, and $\varphi_{0, i}$ in Eq. (2) can be obtained in various ways. Here it is proposed to prescribe one of these parameters and to calculate the other two parameters for given integrated number $\left(N_{i}\right)$ and mass $\left(M_{i}\right)$ concentrations in each section. This approach leads to a self-consistent representation of these quantities in numerical models and also leads to expressions that are relatively straightforward to evaluate.

First, it is useful to introduce the $k$ th moment $\mu_{k i}$ of the size distribution in section $i$, i.e.

$\mu_{k i} \equiv \int_{\varphi_{i-1 / 2}}^{\varphi_{i+1 / 2}} R_{p}^{k} n(\varphi) \mathrm{d} \varphi$.

Using Eqs. (1) and (2) it follows that

$\mu_{k i}=R_{0}^{k} n_{0, i} I_{k i}$,

with

$I_{k i}=\frac{1}{2} \sqrt{\frac{\pi}{\left|\psi_{i}\right|}} f_{k i} \exp \left(k \varphi_{0, i}+\frac{k^{2}}{4\left|\psi_{i}\right|}\right)$,

and

$f_{k i}= \begin{cases}\operatorname{erf}\left(a_{k i+1 / 2}\right)-\operatorname{erf}\left(a_{k i-1 / 2}\right) & \text { if } \psi_{i}>0, \\ \operatorname{erfi}\left(a_{k i+1 / 2}\right)-\operatorname{erfi}\left(a_{k i-1 / 2}\right) & \text { if } \psi_{i}<0 .\end{cases}$

The arguments of the error function $\operatorname{erf}(x)$ and imaginary error function erfi $(x)$ in $f_{k i}$ are given by:

$a_{k i+1 / 2}=-\sqrt{\left|\psi_{i}\right|}\left(\Delta \varphi_{i}-\Delta \varphi_{\star}\right)-\frac{k \operatorname{sgn}\left(\psi_{i}\right)}{2 \sqrt{\left|\psi_{i}\right|}}$,

$a_{k i-1 / 2}=-\sqrt{\left|\psi_{i}\right|} \Delta \varphi_{i}-\frac{k \operatorname{sgn}\left(\psi_{i}\right)}{2 \sqrt{\left|\psi_{i}\right|}}$,

with $\operatorname{sgn}(x)=2 H(x)-1$,

$\Delta \varphi_{i} \equiv \varphi_{0, i}-\varphi_{i-1 / 2}$,

and section size $\Delta \varphi_{\star} \equiv \varphi_{i+1 / 2}-\varphi_{i-1 / 2}$.

Consequently, the integrated number $(k=0)$ and mass $(k=3)$ concentrations in each section are:

$N_{i}=n_{0, i} I_{0 i}$,

$M_{i}=\frac{4 \pi}{3} \rho_{p, i} R_{0}^{3} n_{0, i} I_{3 i}$,

where $\rho_{p, i}$ is the particle density,

As shall be seen, it is useful for the calculation of the fitting parameters to also introduce the dimensionless size ratio

$r_{i} \equiv \frac{\hat{\varphi}_{i}-\varphi_{i-1 / 2}}{\Delta \varphi_{\star}}$

where

$\hat{\varphi}_{i} \equiv \ln \left[\frac{1}{R_{0}}\left(\frac{3}{4 \pi \rho_{p, i}} \frac{M_{i}}{N_{i}}\right)^{1 / 3}\right]$.

is an effective particle size.

$r_{i}$ (Eq. 6) characterizes the skewness of the approximated size distribution within each section. For example, consider two populations of aerosol particles that have the same total 


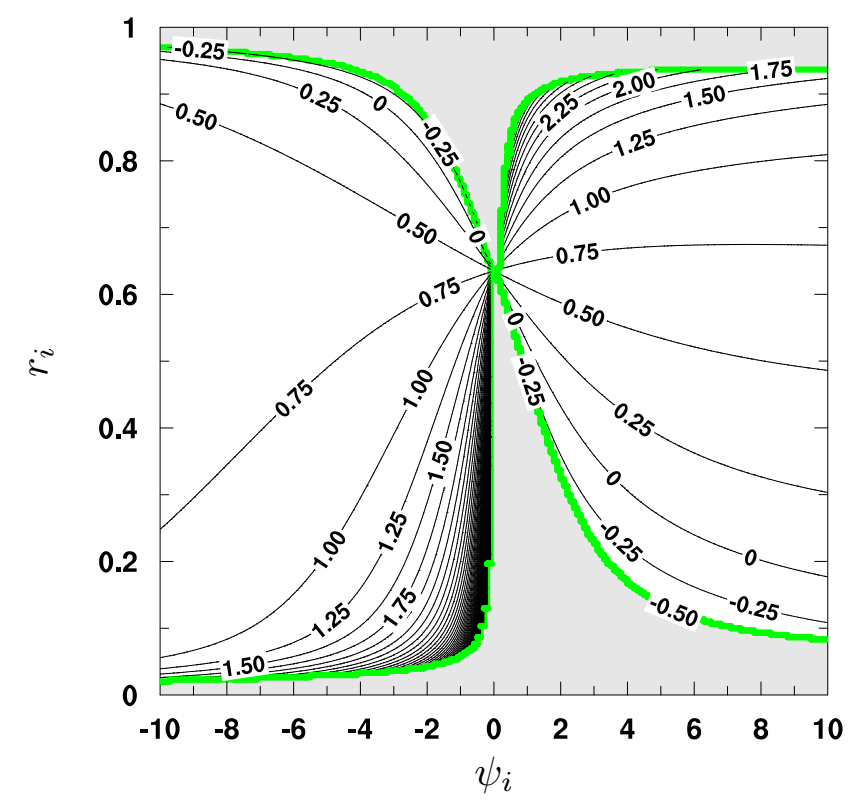

Fig. 1. $\Delta \varphi_{i}$ (Eq. 3) according to Eq. (7). No solution exists in the shaded area.

number of particles but different total mass concentrations within a given range of particle sizes. This can only be the case if the population with the higher mass concentration includes fewer of the small and more of the big particles compared to the other population. Hence, the number size distribution of the former aerosol population is skewed towards larger particle sizes relative to the other number size distribution. Any meaningful values of $N_{i}$ and $M_{i}$ require that $0 \leq r_{i} \leq 1$. Mathematically, the boundary of the manifold that contains the solutions to Eqs. (4) and (5) is given by deltadistributed particle number concentrations for minimum and maximum particle sizes in the given size range (i.e. corresponding to the section boundaries).

Combination of an expression for $M_{i} / N_{i}$ from Eqs. (4) and (5) with Eq. (6) conveniently eliminates $n_{0, i}$, i.e.

$\frac{f_{3 i}}{f_{0 i}}=\exp \left[3\left(r_{i} \Delta \varphi_{\star}-\Delta \varphi_{i}\right)-\frac{9}{4\left|\psi_{i}\right|}\right]$.

The only unknown parameters in this equation are $\Delta \varphi_{i}$ and $\psi_{i}$ if $N_{i}$ and $M_{i}$ and the section boundaries are given. It is suggested to prescribe $\psi_{i}$ so that this equation can be used to determine $\Delta \varphi_{i}$. Subsequently, Eq. (3) can be used to determine the value of the fitting parameter $\varphi_{0, i}$.

An example for $\Delta \varphi_{i}$ from the solution of Eq. (7) is shown in Fig. 1 for a section size $\Delta \varphi_{\star}=\frac{1}{3}[\ln (1.75)-\ln (0.05)]$ (i.e. similar to the application in Sect. 3). Note that Eq. (7) does not have any solution for $\Delta \varphi_{i}$ within a certain range of values of $\psi_{i}$ and $r_{i}$. Therefore, $\psi_{i}$ is selected such that $\psi_{i}$ is equal to an arbitrarily prescribed value $\psi_{m, i}$ inside the region for which a solution of Eq. (7) exists. Outside that region, a different value than that is selected in order to ensure that a

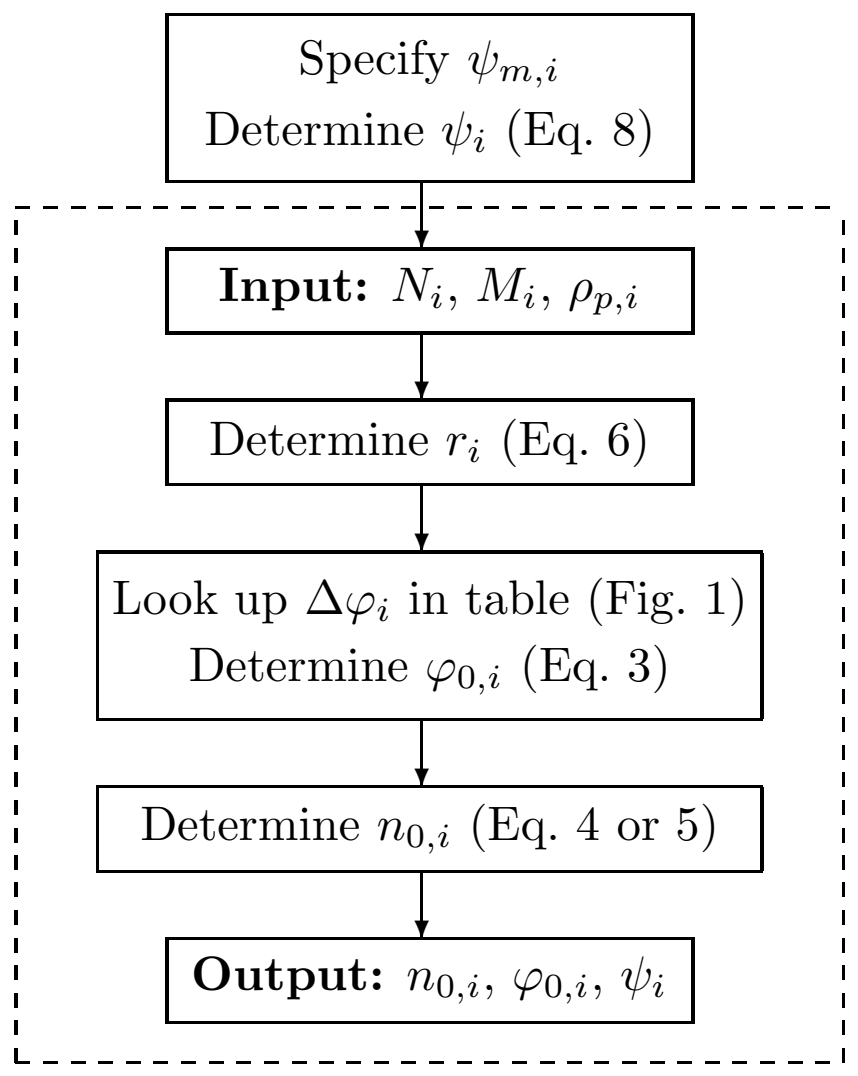

Fig. 2. Summary of the algorithm to determine the fitting parameters in Eq. (2). In applications of the algorithm in models, the steps in the box at the top should be part of the pre-processing stage in the model. The inputs for the other steps may change at each time step in the model and will generally have to be repeated once size distributions are updated in the model.

solution exists. In that case, $\psi_{i}$ should be as close as possible to $\psi_{m, i}$. Therefore,

$\psi_{i}= \begin{cases}\min \left(\psi_{m, i}, \psi_{l}\right) & \text { if } \psi_{m, i}<0, \\ \max \left(\psi_{m, i}, \psi_{l}\right) & \text { if } \psi_{m, i}>0,\end{cases}$

where $\psi_{l}$ represents a threshold for which a solution of Eq. (7) can be found (i.e. as indicated by the green line in Fig. 1). This algorithm can be applied to $0 \leq r_{i} \leq 1$ and $-\infty \leq \psi_{m, i} \leq \infty$ for

$\lim _{r_{i} \rightarrow 0,1} \psi_{l}= \begin{cases}+\infty & \text { if } \psi_{i}>0, \\ -\infty & \text { if } \psi_{i}<0 .\end{cases}$

In practice, it should be sufficient to create a look-up table for $\Delta \varphi_{i}$ as a function of $\psi_{i}$ and $r_{i}$ and to use this result to obtain the complete set of fitting parameters from Eqs. (3) and (4) or (5). Although not necessary, it is convenient to assume that all sections have the same size so that only one look-up table is required.

The algorithm described above is summarized in Fig. 2. It provides the fitting parameters for each section in Eq. (2) for 
given values of the number and mass concentrations in the same section.

While the accuracy of the approximation of a size distribution in terms of log-normal size distributions according to Eq. (2) depends on the values of $\psi_{m, i}$, no attempt is made here to calculate $\psi_{m, i}$ as part of the algorithm. Tests with the method under various conditions give evidence for a relatively weak dependency of the errors of the method on $\psi_{m, i}$ within a substantial range for this parameter (e.g., Sects. 3 and 5).

The accuracy of the transformations from $N_{i}$ and $M_{i}$ to log-normal size distributions according to the algorithm (Fig. 2) and vice versa (Eqs. 4 and 5) depends on the accuracy of the tabulated data for $\Delta \varphi_{i}$. However, for typical applications, (e.g. in the examples presented in the following sections) sufficient accuracy can usually be achieved for reasonably small table sizes. If necessary, the accuracy can be additionally increased by directly solving Eq. (7) instead of (or in addition to) the table look-up step.

It should be noted here that similar to the approach proposed here, Zender et al. (2003) proposed a piecewiseanalytical representation of the aerosol size distribution. Specifically, they assumed that the aerosol mass is lognormally distributed in each section. However, their approach is quite similar to the single-moment sectional approach. In particular, the assumption of time-invariant size distributions within each section, with the mass being the only predicted variable, clearly distinguishes their approach from the PLA method. Consequently, aerosol number (or any other moment) will generally not be conserved in simulations with Zender et al.'s approach. Additionally, the assumption of a time-invariant size distribution could be quite problematic at small to moderate numbers of sections. For example, this assumption cannot be expected to not work well for gravitational settling of mineral dust particles which typically causes large variations in the particle size distribution with height. In contrast to Zender et al.'s approach, the PLA method can be used to account for changes in the size distribution at any given particle size scale.

\subsection{Computational costs of the method}

For applications of the PLA method in numerical models it is useful to consider the computational costs that are potentially associated with the method. In a model, the calculations of $r_{i}$ and $n_{0, i}$ in Fig. 2 would likely be the most expensive steps because of the need to evaluate the functions $\ln (x)$ and $\operatorname{erf}(x)$ [respectively erfi $(x)$ ] for each section according to Eqs. (6) and (4). In comparison, the costs associated with the table look-up are relatively minor for a typical Fortran 90-implementation on an IBM pSeries 390 system with POWER4 microprocessor architecture at the Canadian Meteorological Centre (i.e. $\approx 1 / 3$ of the total costs of the method). These costs are considerably smaller than the costs that would typically be associated with parameterizations of bulk aerosol processes in atmospheric models.

\section{Application to observed size distributions}

As a first test of the PLA method, the method was used to approximate aerosol size distributions that are based on observational results from the New York City (NYC) Urban Plume Experiment that took place between 1 to 28 July 1996 (Kleinman et al., 2000). In this experiment, aerosol number size distributions were determined from an aircraft that was equipped with a Passive Cavity Aerosol Spectrometer Probe (PCASP) with 15 particle size classes in the diameter range from $D_{p}=0.1$ to $3.5 \mu \mathrm{m}$. The PCASP dries the sampled aerosol by heating so that the aerosol size distributions are representative for solid aerosol particles.

The observed number size distributions were first fitted using cubic interpolating splines for each one of the 7818 samples, yielding continuous number size distributions $(\mathrm{d} N / \mathrm{d} \varphi)_{\text {spl }}$. Secondly, $(\mathrm{d} N / \mathrm{d} \varphi)_{\text {spl }}$ was used to calculate the corresponding mass size distribution for an arbitrary particle density of $\rho_{p}=1 \mathrm{~g} \mathrm{~cm}^{-3}$. Average results for the original data and interpolated number and mass size distributions for this experiment are shown in Fig. 3. In the following, they serve as a reference for comparisons with the PLA and other methods for a particle diameter range from 0.1 to $3.5 \mu \mathrm{m}$.

For the applications described in the following, $(\mathrm{d} N / \mathrm{d} \varphi)_{\text {spl }}$ was integrated over each section to determine number and mass concentrations

$\begin{aligned} N_{i} & =\int_{\varphi_{i-1 / 2}}^{\varphi_{i+1 / 2}}\left(\frac{\mathrm{d} N}{\mathrm{~d} \varphi}\right)_{\mathrm{spl}} \mathrm{d} \varphi, \\ M_{i} & =\frac{4 \pi}{3} \rho_{p} \int_{\varphi_{i-1 / 2}}^{\varphi_{i+1 / 2}} R_{p}^{3}\left(\frac{\mathrm{d} N}{\mathrm{~d} \varphi}\right)_{\mathrm{spl}} \mathrm{d} \varphi,\end{aligned}$

$N_{i}$ and $M_{i}$ were then used to obtain the fitting parameters in Eq. (2) according to the procedure described in Sect. 2.2 for the PLA method. The corresponding results are indicated by red lines in Fig. 3.

Additionally, the single-moment bin approach (hereafter referred to as SMB) was used to obtain number and mass size distributions from

$$
\begin{aligned}
& \left(\frac{\mathrm{d} N}{\mathrm{~d} \varphi}\right)_{\mathrm{SMB}, i}=\frac{N_{i}}{\Delta \varphi_{\star}}, \\
& \left(\frac{\mathrm{d} M}{\mathrm{~d} \varphi}\right)_{\mathrm{SMB}, i}=\frac{M_{i}}{\Delta \varphi_{\star}}
\end{aligned}
$$

(dark blue lines in Figs. 3a and b). As an alternative to $(\mathrm{d} M / \mathrm{d} \varphi)_{\mathrm{SMB}, i}$, the mass size distribution has also been diagnosed based on the number size distribution, i.e.

$\left(\frac{\mathrm{d} M}{\mathrm{~d} \varphi}\right)_{\mathrm{mSMB}, i}=\frac{4 \pi}{3} \rho_{p} R_{c, i}^{3}\left(\frac{\mathrm{d} N}{\mathrm{~d} \varphi}\right)_{\mathrm{SMB}, i}$,

where $R_{c, i}$ is the radius in the centre of the sections (green line in Fig. 3b). 
(a)

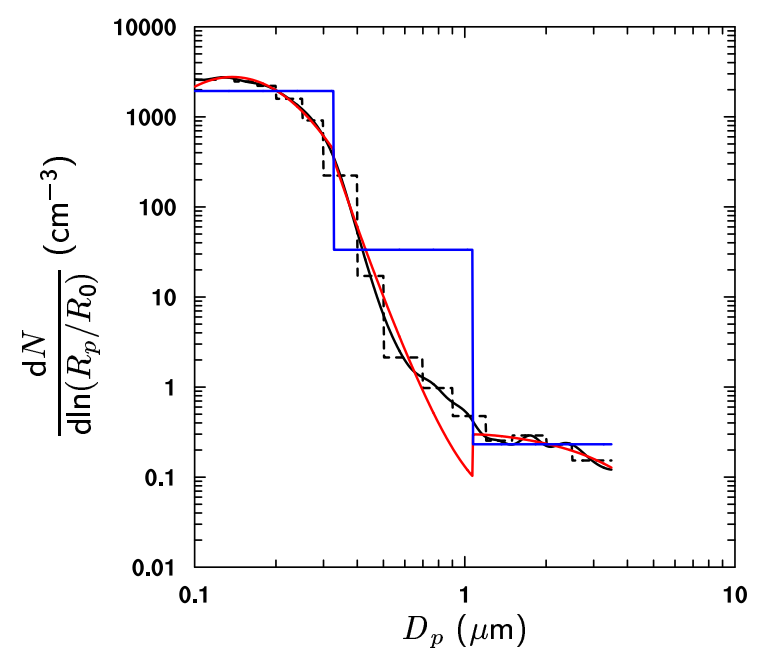

(b)

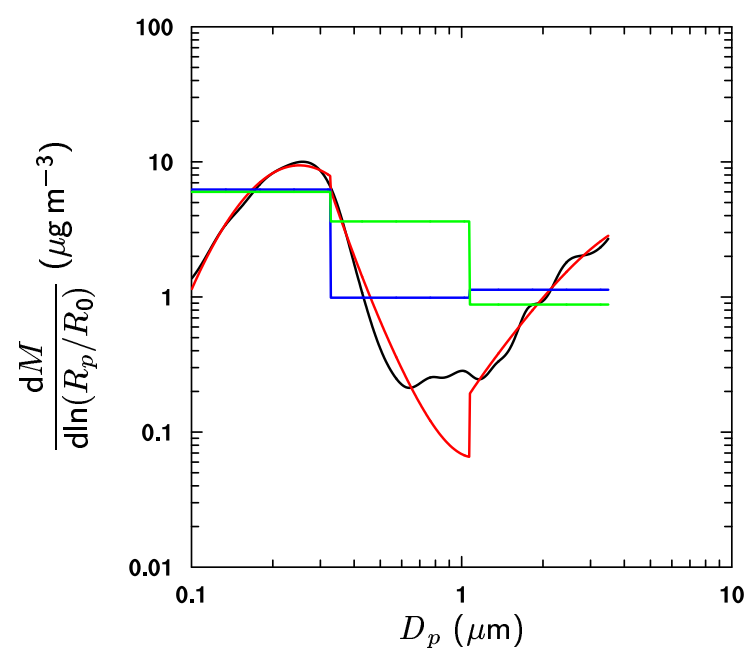

Fig. 3. Average observed (dashed line) and interpolated size distributions (full black lines) for aerosol number (left column) and mass (right column) for all samples during the NYC Urban Plume Experiment. Red lines refer to the corresponding results of the PLA method for 3 sections. Similar, dark blue lines in (a) and (b) refer to results of the single-moment bin (SMB) approach. The green line in (b) is for a diagnosed size distribution based on results of the bin method in (a) as described in the text.

Results obtained with the PLA method are much closer to the reference size distribution in comparison to the SMB approach in Figs. 3a and b. Although both approaches produce the same mean number and mass concentrations for each section (per definition), the statistical properties of the size distributions are much better captured with the PLA method. Furthermore, the SMB approach leads to significantly biased results for the mass size distribution if the integrated number concentration in each section is the only available statistical information for the fit (green line in Fig. 3). This illustrates that it may be problematic to use a diagnostic relationship between aerosol number and mass size distributions in an aerosol model if the size distribution is not well resolved.

Figure 4 shows root mean square (rms) differences between the approximated and spline interpolated size distributions as a function of the number of sections for the comparison in Fig. 3. For the PLA method, $\psi_{m, i}$ (Eq. 8) was determined by minimizing the rms differences $\left(\psi_{m, i}=2.9,-0.2,1.8\right)$. Additional tests with $\psi_{m, i}=1$ and $\psi_{m, i}=4$ for all sections are also shown in the figure.

The rms differences for the PLA method (red lines) with the various choices for $\psi_{m, i}$ are systematically lower than those for the SMB approach (dark blue lines), especially at larger number of sections. The PLA method for 3 sections produces roughly the same rms differences as the SMB approach for 10 sections. When 15 sections are employed for the PLA method, about 7 times the number of sections is required to obtain the same accuracy in the SMB approach. Errors caused by non-optimal choices for $\psi_{m, i}$ for the PLA method are small compared to the errors that are associated with the SMB approach.
In order to put results of the PLA method into further perspective, the double-moment bin approach proposed by Tzivion et al. (1987) (hereafter referred to as TFL) has also been used. Based on this approach, number and mass size distributions in each section can be expressed as linear functions of the particle mass $m_{p}=\frac{4 \pi}{3} \rho_{p} R_{p}^{3}$, i.e.

$$
\begin{aligned}
\left(\frac{\mathrm{d} N}{\mathrm{~d} m_{p}}\right)_{\mathrm{TFL}, i} & =a_{i}\left(\frac{m_{p, i+1 / 2}-m_{p}}{\Delta m_{p, i}}\right) \\
& +b_{i}\left(\frac{m_{p}-m_{p, i-1 / 2}}{\Delta m_{p, i}}\right), \\
\left(\frac{\mathrm{d} M}{\mathrm{~d} m_{p}}\right)_{\mathrm{TFL}, i} & =m_{p, i-1 / 2} a_{i}\left(\frac{m_{p, i+1 / 2}-m_{p}}{\Delta m_{p, i}}\right) \\
& +m_{p, i+1 / 2} b_{i}\left(\frac{m_{p}-m_{p, i-1 / 2}}{\Delta m_{p, i}}\right),
\end{aligned}
$$

with $\Delta m_{p, i}=m_{p, i+1 / 2}-m_{p, i-1 / 2}$. Although the original method does not require that the points at the edges of the sections are collocated in Eqs. (11) and (12), this assumption will be employed here for the sake of a more straightforward comparison of the basic assumptions in the different schemes and their impact on simulation results.

The coefficients $a_{i}$ and $b_{i}$ in Eqs. (11) and (12) can be obtained from integration over $\left(\mathrm{d} N / \mathrm{d} m_{p}\right)_{\mathrm{TFL}, i}$ and $\left(\mathrm{d} M / \mathrm{d} m_{p}\right)_{\mathrm{TFL}, i}$ and using $N_{i}$ and $M_{i}$ from Eqs. (9) and (10).

Somewhat surprisingly, results for TFL's approach (light blue lines in Figs. 4a and b) are systematically worse than those for the SMB method. The reason for the large differences is that TFL compute size distributions based on $m_{p}$ 
(a)

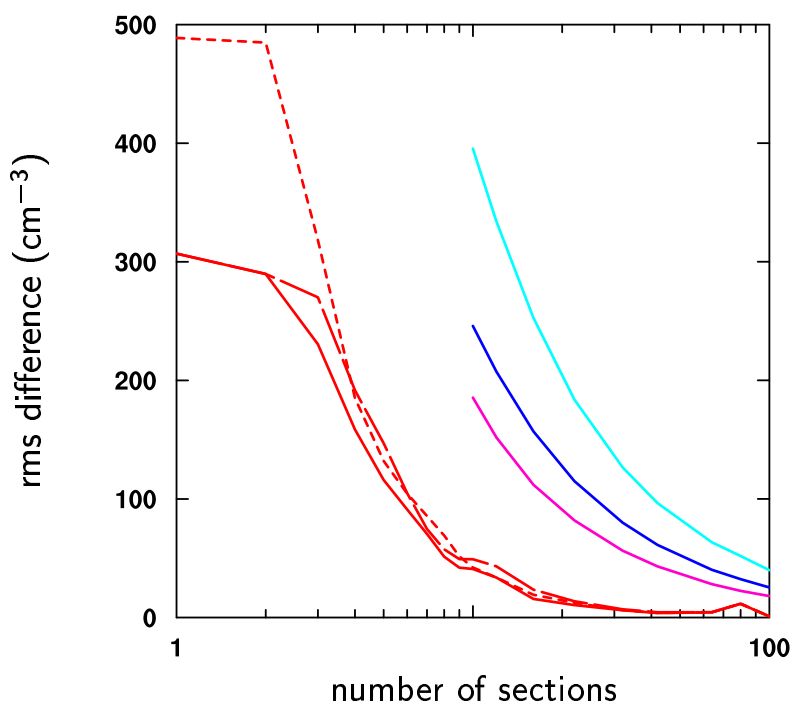

(c)

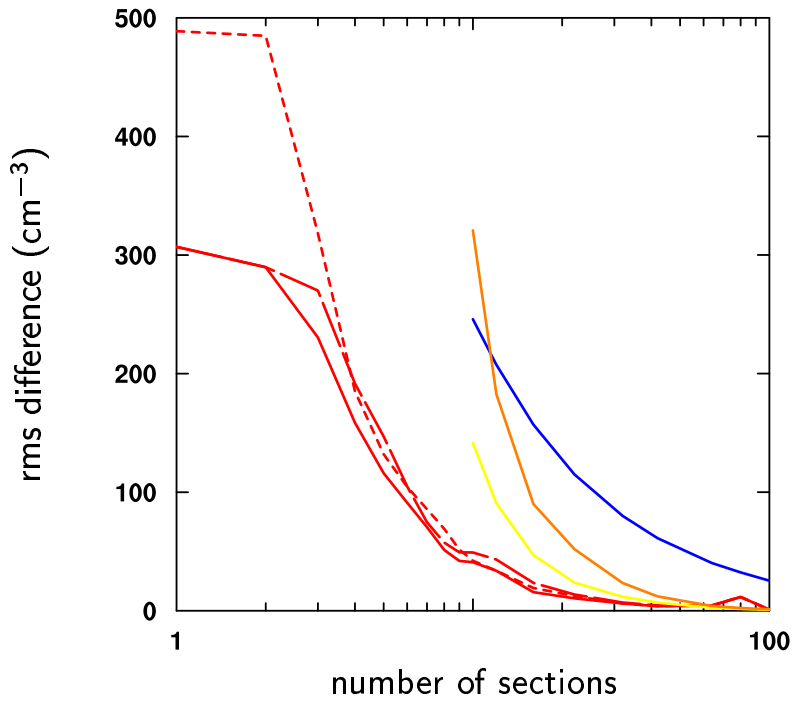

(b)

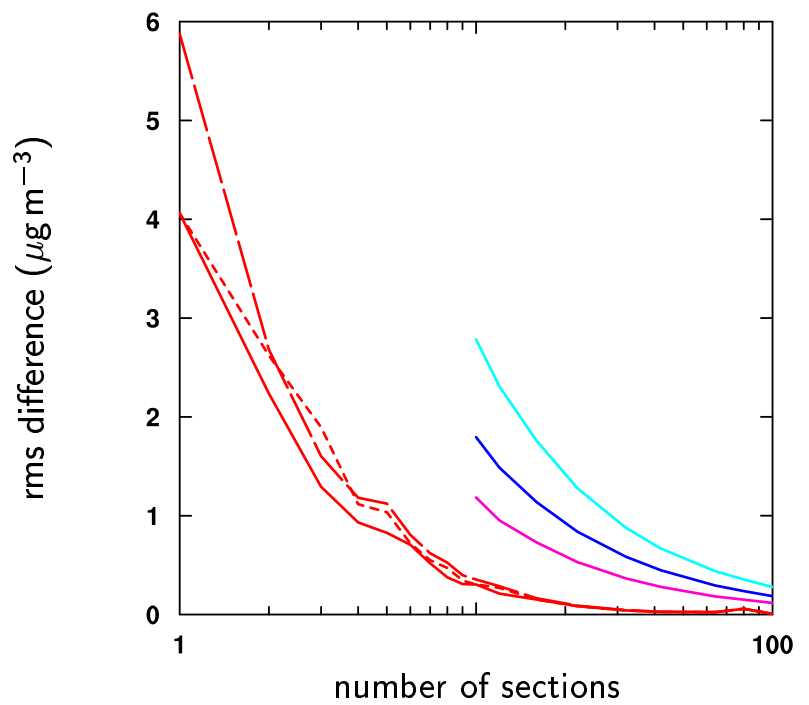

(d)

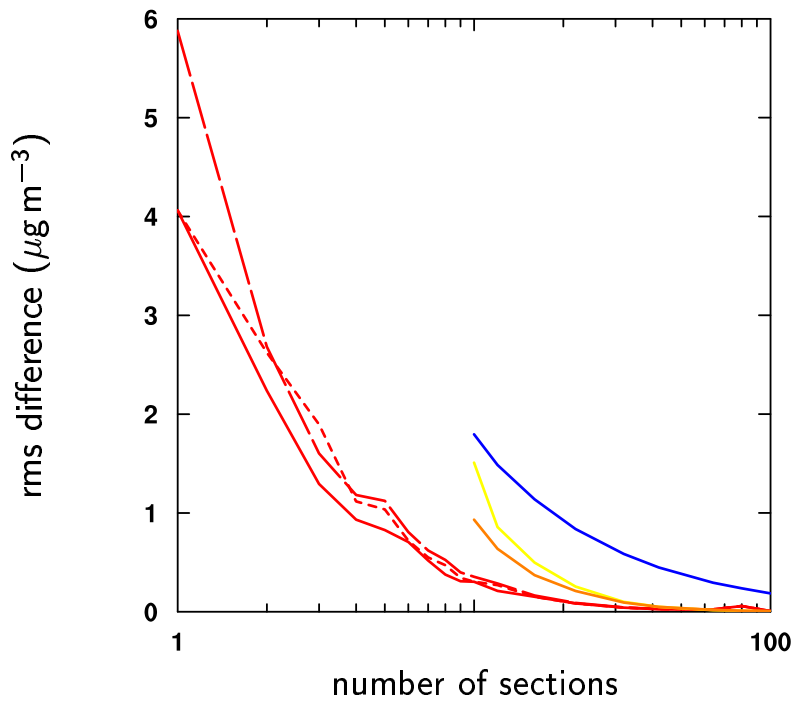

Fig. 4. Mean rms differences between the approximated and spline interpolated size distributions for aerosol number (a and c) and mass (b and d) for all samples from the NYC experiment. Dark blue lines refer to the single-moment bin approach and red lines to the PLA method for optimal choice of $\psi_{m, i}$ (full line), $\psi_{m, i}=1$ (long dashed line), and $\psi_{m, i}=4$ (short dashed line). In (a) and (b), light blue lines refer to the approach by Tzivion et al. (1987) and pink lines to the modified version of this approach. (c) and (d) show results for the polynomial approximations based on the number (yellow lines) and mass (orange lines) size distributions. Only results for less than doubling of mass between section boundaries are considered for the other methods because these methods are typically not employed for broader sections.

rather than on $\varphi$. This approach is problematic in the context of the observed size distributions from the NYC Urban Plume Experiment because $\left(\mathrm{d} N / \mathrm{d} m_{p}\right)_{\text {spl }}$ is much steeper than $(\mathrm{d} N / \mathrm{d} \varphi)_{\mathrm{spl}}$. It can be analytically shown that for any given linear number distribution $\mathrm{d} N / \mathrm{d} m_{p}$, the piecewiselinear approximation of $\mathrm{d} N / \mathrm{d} m_{p}$ according to TFL's approach systematically produces smaller absolute values for the slopes of the distribution in each section.

The results for TFL's approach demonstrate that it is important to consider the same particle size variable when com- paring results for different approximation methods. For a fairer comparison, TFL's approach has been modified in order to express the approximated number and mass size distributions as linear functions of $\varphi$ instead, i.e.

$$
\begin{aligned}
\left(\frac{\mathrm{d} N}{\mathrm{~d} \varphi}\right)_{\mathrm{mTFL}, i} & =a_{m, i}\left(\frac{\varphi_{i+1 / 2}-\varphi}{\Delta \varphi_{\star}}\right) \\
& +b_{m, i}\left(\frac{\varphi-\varphi_{i-1 / 2}}{\Delta \varphi_{\star}}\right),
\end{aligned}
$$




$$
\begin{aligned}
\left(\frac{\mathrm{d} M}{\mathrm{~d} \varphi}\right)_{\mathrm{mTFL}, i} & =m_{p, i-1 / 2} a_{m, i}\left(\frac{\varphi_{i+1 / 2}-\varphi}{\Delta \varphi_{\star}}\right) \\
& +m_{p, i+1 / 2} b_{m, i}\left(\frac{\varphi-\varphi_{i-1 / 2}}{\Delta \varphi_{\star}}\right),
\end{aligned}
$$

where, in analogy to the original approach, coefficients $a_{m, i}$ and $b_{m, i}$ can be obtained from integration.

The modified version of this approach (pink lines) yields systematically better performance than the SMB approach according to Figs. $4 a$ and $b$. However, neither the original nor the modified version of TFL's approach outperform the PLA method at any given number of sections.

Finally, it is also interesting to compare the PLA method with a representation of the aerosol size distribution in terms of polynomials. For example, Dhaniyala and Wexler (1996) and von Salzen and Schlünzen (1999a) have used secondorder polynomials to represent aerosol mass size distributions following the approach that was originally proposed by Bott (1989). An interesting feature of Bott's approach is that it ensures conservation from renormalization of the fitted size distribution. However, similar to other single-moment schemes, other moments of the size distribution can only be obtained from diagnostic relationships.

Figures $4 \mathrm{c}$ and $\mathrm{d}$ show results from applications of the polynomial approach to the number (yellow lines) and mass (orange lines) size distributions from the NYC Urban Plume Experiment based on second-order polynomials. For simplicity and lack of a better approach, the corresponding mass (yellow line in Fig. 4d) and number (orange line in Fig. 4c) size distributions have been diagnosed by scaling of the approximated size distributions with $m_{p}$, respectively $1 / m_{p}$. Note that this approach does not ensure conservation for the diagnosed quantities.

The polynomial approach gives very good results when applied to the observed size distributions for 20 or more sections for both versions of this approach and the diagnosed quantities. However, the rms differences are considerable for the diagnosed number size distribution at smaller number of sections if the method is applied to the mass size distribution (and vice versa).

A more substantial problem with the polynomial approach is that it produced negative values of the size distribution for some of the sections in the simulations. Although there are techniques that can be used to prevent negative results in applications of the method to tracer advection (Bott, 1989) and coagulation (Bott, 1998), it does not seem straightforward to come up with a general correction method.

Note that the polynomial approach will no longer be considered in the following owing to the lack of a general correction method for negative values which would be required for the development of parameterizations for gravitational settling and other aerosol dynamical processes.

\section{Application to particle growth by condensation}

The results from the previous section give evidence that the PLA method can be used to accurately approximate atmospheric aerosol size distributions. However, a more important question from a practical point of view is whether the method is also appropriate for modelling individual aerosol dynamical processes. Owing to the considerable number of relevant aerosol dynamical processes and the wide range of atmospheric conditions that influence aerosols size distributions in the atmosphere, an impractically large number of tests would be required to address this question in a sufficiently general way. A detailed study on the performance of the scheme in the context of different aerosol dynamical processes is outside the scope of this paper. Instead, a single application of the method to the simulation of particle growth by condensation is discussed in the following. Several other authors have studied solutions of the condensation equation in the past to test the performance of different numerical schemes. The advantage of this approach is that relatively simple analytical solutions of the condensation equation are available for comparisons with numerical solutions under certain idealized circumstances.

A simple analytical solution of the condensation equation under the assumption of continuum regime growth, unity accommodation coefficient, and constant gas-phase supersaturation was presented by Seinfeld and Pandis (1998). Under these assumptions, the time evolution of the aerosol particle diameter is given by

$\frac{\mathrm{d} D_{p}}{\mathrm{~d} t}=\frac{A_{g}}{D_{p}}$,

with

$A_{g}=\left(\frac{4 D C}{\rho_{p}}\right)$,

the concentration $C$ of the gas (in $\mathrm{kg} \mathrm{m}^{-3}$ ), and its diffusivity $D\left(\right.$ in $\left.\mathrm{m}^{2} \mathrm{~s}^{-1}\right)$.

Application of Eq. (15) to an initial size distribution,

$\frac{\mathrm{d} N}{\mathrm{~d} \varphi}(t=0)=D_{p} \frac{N_{0}}{\sqrt{2 \pi} \ln \sigma} \exp \left[-\frac{\ln ^{2}\left(D_{p} / D_{p g}\right)}{2 \ln ^{2} \sigma}\right]$,

for constant $N_{0}, \sigma$, and $D_{p g}$ yields the solution

$\frac{\mathrm{d} N}{\mathrm{~d} \varphi}(t)=\frac{D_{p}^{2}}{\left(D_{p}^{2}-2 A_{g} t\right)^{\frac{1}{2}}} \frac{N}{\sqrt{2 \pi} \ln \sigma}$
$\exp \left\{-\frac{\ln ^{2}\left[\left(D_{p}^{2}-2 A_{g} t\right)^{\frac{1}{2}} / D_{p g}\right]}{2 \ln ^{2} \sigma}\right\}$.

Results for Eq. (16) are shown in Fig. 5 for the case that was studied by Seinfeld and Pandis (1998)(black lines). 
(a)

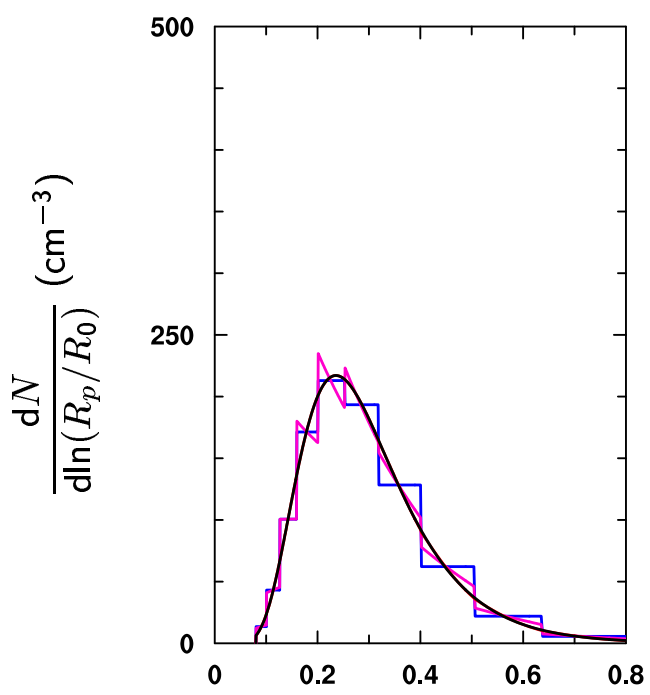

(b)

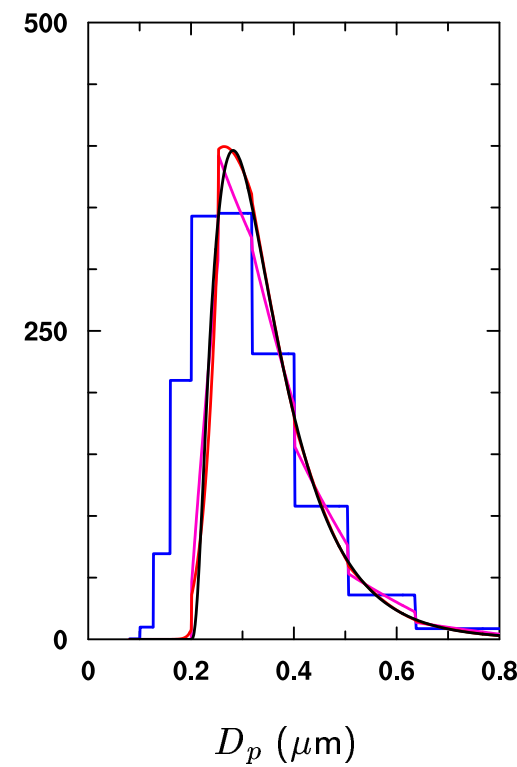

(c)

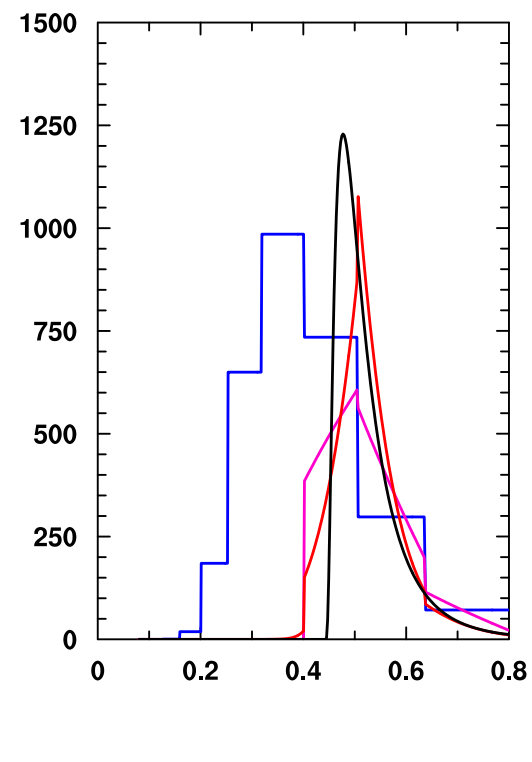

Fig. 5. Size distributions for analytical and numerical solutions of the condensation equation at times $t=0$ (a), $t=2 \mathrm{~min}$ (b), and $t=10 \mathrm{~min}$ (c). Results are shown for the analytical solution (black), PLA (red), SMB (blue), and modified TFL (pink) methods, for $C=4.09 \mu \mathrm{g} \mathrm{m}{ }^{-3}$ (1 ppb), $D_{p g}=0.2 \mu \mathrm{m}, \sigma=1.5, N_{0}=1.01635 \cdot 10^{15} \mathrm{~m}^{-4}, D=0.1 \cdot 10^{-4} \mathrm{~m}^{2} \mathrm{~s}^{-1}$, molecular weight $M_{s}=0.1 \cdot 10^{-3} \mathrm{~kg} \mathrm{~mol}-1, \rho_{p}=1 \cdot 10^{3} \mathrm{~kg} \mathrm{~m}{ }^{-3}$, $T=298 \mathrm{~K}, P=1013.25 \mathrm{hPa}$. The time step is $\Delta t=1 \mathrm{~s}$. For application of the PLA method, $\psi_{m, i}=3$ for all sections.

Parameterizations for condensational growth for PLA, SMB, and modified TFL methods are based on simplified versions of the parameterizations that are described in Appendix A (which are for applications in atmospheric models as described in Sect. 5). Specifically, treatments of nucleation, non-continuum effects, non-ideal accommodation, and time-dependent gas-phase concentrations were removed from the parameterizations so that they can be applied in the current study. For results shown in Fig. 5, 10 sections in the range from $D_{p}=0.08 \mu \mathrm{m}$ to $0.8 \mu \mathrm{m}$ were used, which approximately corresponds to a doubling in particle mass between the section boundaries.

The simulations were initialized based on integrated mass and number concentrations over the ranges of the sections. The initial conditions (time $t=0$ ) are shown in Fig. 5. For each method, the initial size distribution is reasonably well represented. Although there are some differences between the initial size distributions, the effects on the sectionintegrated growth rates are rather small during the first time steps of the simulations.

The time evolution of the number size distribution is very well captured in the simulations with the PLA and modified TFL methods in Fig. 5b. However, the steep slope at the trailing end of the size distribution at $t=10 \mathrm{~min}$ (Fig. 5c) bis not well represented because of the largely unresolved features of the size distribution at that time for the given section sizes.
Results of the SMB approach are clearly deficient in Fig. 5. With this approach, the particle growth is not nearly as efficient for the small particles as required. At $t=10 \mathrm{~min}$, the simulated size distribution is substantially broader than the analytical solution. Additionally, the total particle number concentration increases over time in the simulation with the SMB approach. The reason for the non-conservation of particle number concentration is that the parameterization for the SMB approach is based on particle mass as the prognostic variable. The particle number concentration is determined diagnostically (see Appendix A3 for details). Such a treatment is common practice in applications of the singlemoment bin approach to aerosol modelling.

An analysis of the rms errors of the simulated size distributions relative to the analytical solution confirms that the quality of the results of the PLA and modified TFL methods is comparable for simulations with 10 sections (Fig. 6).

Tests for various numbers of sections yield some characteristic features of the different methods. At $t=0$, the behaviour of the rms errors is similar to the results in Fig. 4. For all methods, the rms errors increase noticeably over the course of the simulations. The errors become quite substantial at $t=10 \mathrm{~min}$. At that time, most of the features of the size distribution cannot be resolved so that none of the methods performs particularly well.

Results of the SMB method are considerably less accurate compared to results of the PLA and TLF method, even for simulations with 100 sections. 
(a)

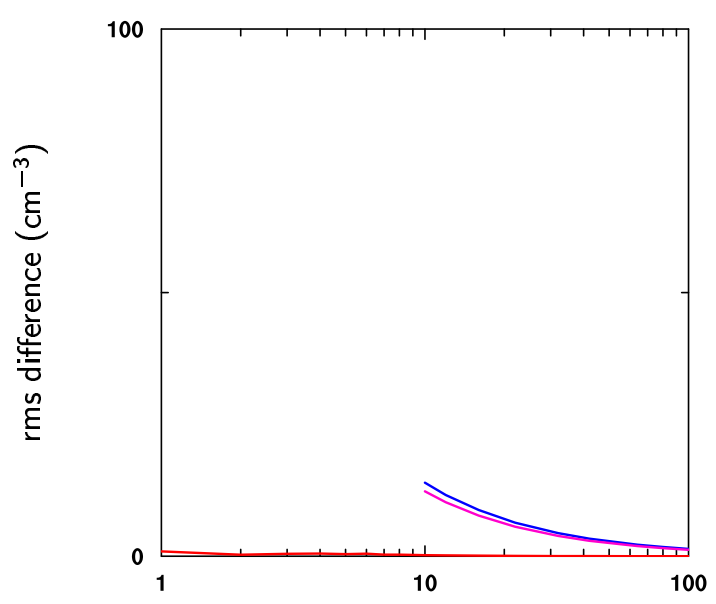

(c)

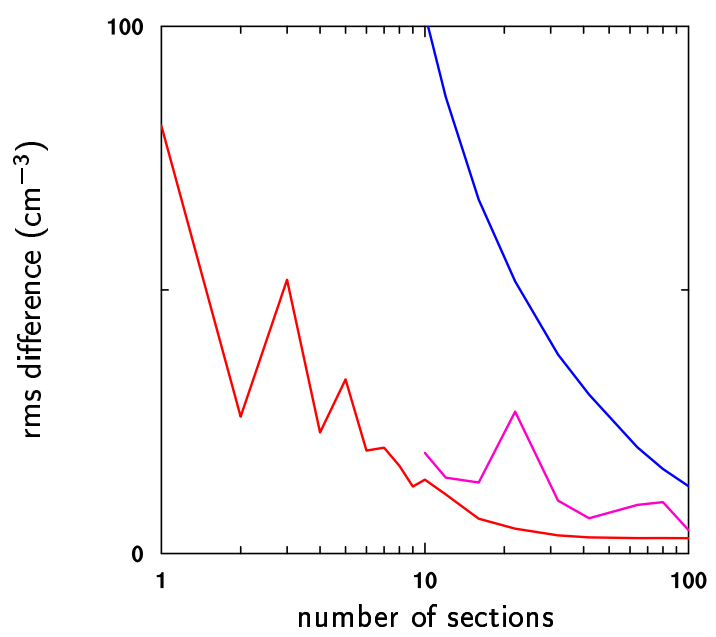

(e)

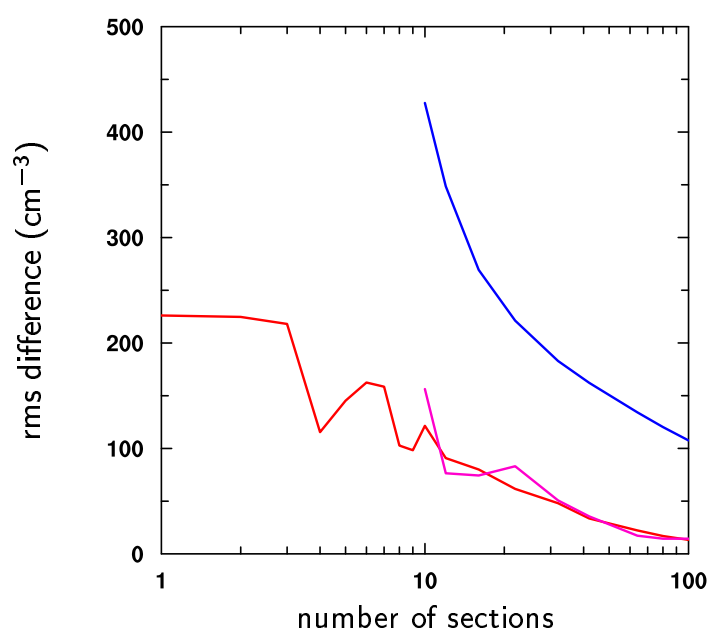

(b)

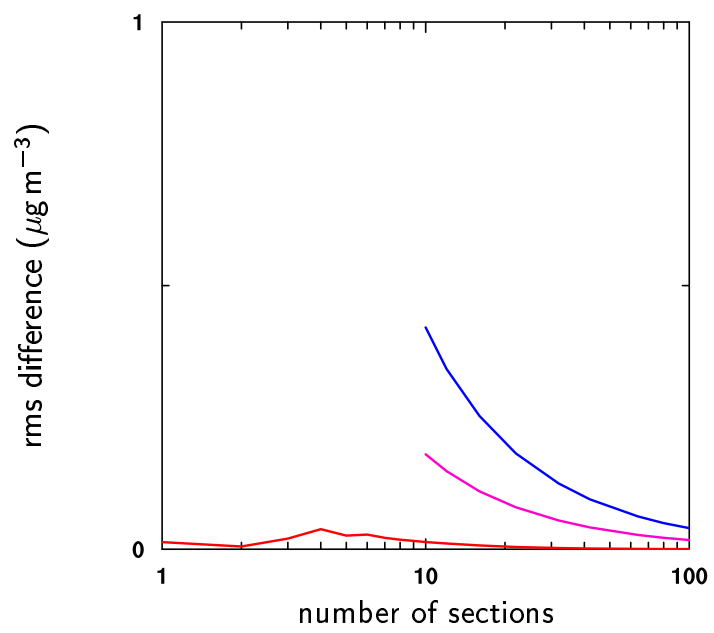

(d)

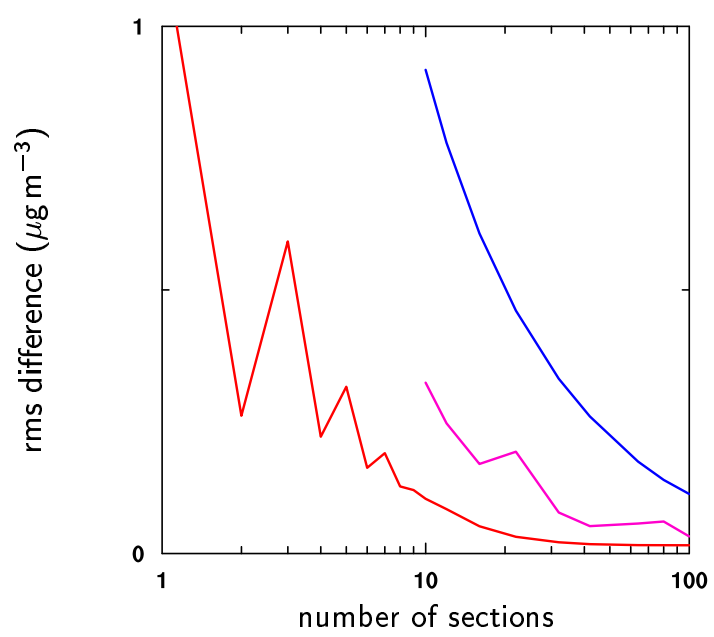

(f)

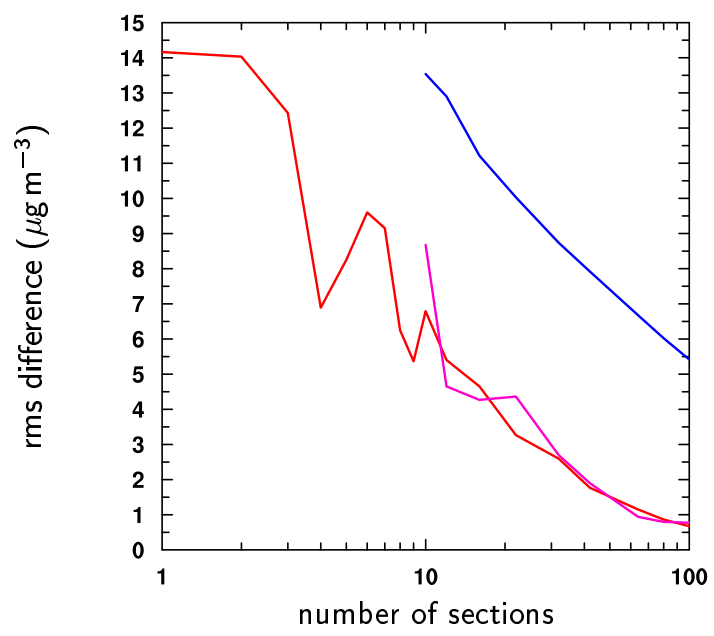

Fig. 6. Rms differences between simulated and analytical size distributions for aerosol number (a, $\mathbf{c}$, and $\mathbf{e})$ and mass $(\mathbf{b}, \mathbf{d}$, and $\mathbf{f})$. (a) and (b) are for $t=0$, (c) and (d) for $t=2 \mathrm{~min}$, and (e) and (f) for $t=10 \mathrm{~min}$. The colours correspond to different numerical methods, similar to Fig. 5. 
At $t=2$ min, the PLA method produces noticeably lower rms errors than the SMB or modified TFL approaches for most of the simulations. However, the rms errors vary substantially for different number of sections for the PLA and TLF methods. The fact that no simple relationship exists between the rms errors and size resolution makes it difficult to draw firm conclusions on the relative accuracy of the methods with respect to each other. However, from the results in Fig. 6, results for 4 or 5 sections with the PLA method can probably be expected to be not much less accurate than results for 10 or more sections with the modified TFL method in applications of the method to the condensation equation.

A time step of $\Delta t=1 \mathrm{~s}$ was used to generate the results in Fig. 6. This choice ensures that the maximum Courant numbers for the increases in particle size per time step do not exceed a critical threshold at high size resolutions in the simulations. However, the maximum Courant numbers are approximately proportional to the number of sections in the simulations so that simulations with lower number of sections do not require time steps as low as $\Delta t=1 \mathrm{~s}$ for sufficiently accurate results. For example, the maximum Courant number is $C r=0.044$ in the simulation with the PLA method for 4 sections in Fig. 6. The corresponding rms errors at $t=2 \mathrm{~min}$ are $23 \mathrm{~cm}^{-3}$ and $0.22 \mu \mathrm{g} \mathrm{m}^{-3}$ for the number and mass size distributions, respectively. An additional simulation for the same case but with a time step of $\Delta t=30 \mathrm{~s}$ yields a maximum Courant number of $C r=0.99$ and rms errors of $27 \mathrm{~cm}^{-3}$ and $0.24 \mu \mathrm{g} \mathrm{m}^{-3}$ for the number and mass size distributions, respectively. Hence, the combined benefits of a low number of sections and large time steps can potentially lead to much more efficient simulations with the PLA method compared to the other methods.

\section{Single-column model simulations}

In the literature, parameterizations of aerosol dynamical processes are often tested on an individual basis and only for very specific cases. There is no agreed protocol that would allow to put results of these studies into the context of general situations that occur in the atmosphere. On the other hand, interactions between different processes can be studied with coupled atmosphere/aerosol models under more realistic conditions.

In the following, parameterizations for nucleation (i.e. the formation of new particles from the gas-phase), condensation of sulphuric acid $\left(\mathrm{H}_{2} \mathrm{SO}_{4}\right)$, and gravitational settling are tested in the context of the CCCma atmospheric single column model (SCM). These processes are strongly dependent upon particle size so that simulations of these processes are sensitive to the numerical representation of the particle size distribution. The application of a numerical approach to nucleation and condensation constitutes a strong test of the approach owing to the considerable degree of non-linearity that arises from the competing effects of nucleation and conden- sation for the gas-to-particle transfer of mass. Among others, the accuracy of solutions for these processes depends strongly on the ability of the algorithm to faithfully represent the condensation driven transport of particle properties over a wide range of sizes in the particle spectrum.

The parameterizations for nucleation, condensation, and gravitational settling are described in the Appendix. The basic physical and chemical equations have been discretized using the PLA, SMB, and modified TFL methods (as described in the previous sections). Different numerical techniques are used for the methods depending on the different information that is available about the aerosol size distribution from each method.

\subsection{Model description}

The aerosol parameterizations have been implemented in the latest version of the CCCma single column model (SCM4). This model uses the same physics and chemistry parameterizations as in CCCma's fourth generation AGCM (von Salzen et al., 2005). The aerosol concentrations, i.e. $M_{i}$ (for the PLA, modified TFL, and SMB methods) and $N_{i}$ (for the PLA and modified TFL methods), are carried as fully prognostic tracers in SCM4.

The model domain extends from the surface up to the stratopause region $(1 \mathrm{hPa}$, approximately $50 \mathrm{~km}$ above the surface). This region is spanned by 35 layers. The mid point of the lowest layer is approximately $50 \mathrm{~m}$ above the surface at sea level. Layer depths increase monotonically with height from approximately $100 \mathrm{~m}$ at the surface to $3 \mathrm{~km}$ in the lower stratosphere. The vertical discretization is in terms of rectangular finite elements defined for a hybrid vertical coordinate as described by Laprise and Girard (1990). Although the SCM does not resolve any processes that occur in the horizontal direction, results are meant to represent the mean situation in an area similar in size to a typical AGCM grid cell, e.g. for horizontal grid sizes on the order of hundreds of kilometers.

The model time step in SCM4 is $20 \mathrm{~min}$. The discretization in time is based on the leapfrog time stepping method. The subroutines for aerosol dynamical processes use the same time step and are therefore called once per model time step. For the PLA method, the conversion of $N_{i}$ and $M_{i}$ to the fitting parameters $n_{0, i}, \psi_{i}$, and $\varphi_{0, i}$ according to the procedure as outlined in Fig. 2 is done before each subroutine that requires the fitting parameters as input (i.e. twice per model time step). Similar, fitting parameters $a_{m, i}$ and $b_{m, i}$ in Eqs. (13) and (14) are obtained with the same frequency in simulations with the modified TFL approach.

In addition to the newly introduced aerosol dynamical processes, the aerosol tracers are subject to vertical transport and wet deposition in the model. For simplicity, wet deposition is treated as a size-independent process based on the parameterizations that are available for bulk sulphate aerosol in SCM4 (Lohmann et al., 1999; von Salzen et al., 2000). 
(a)

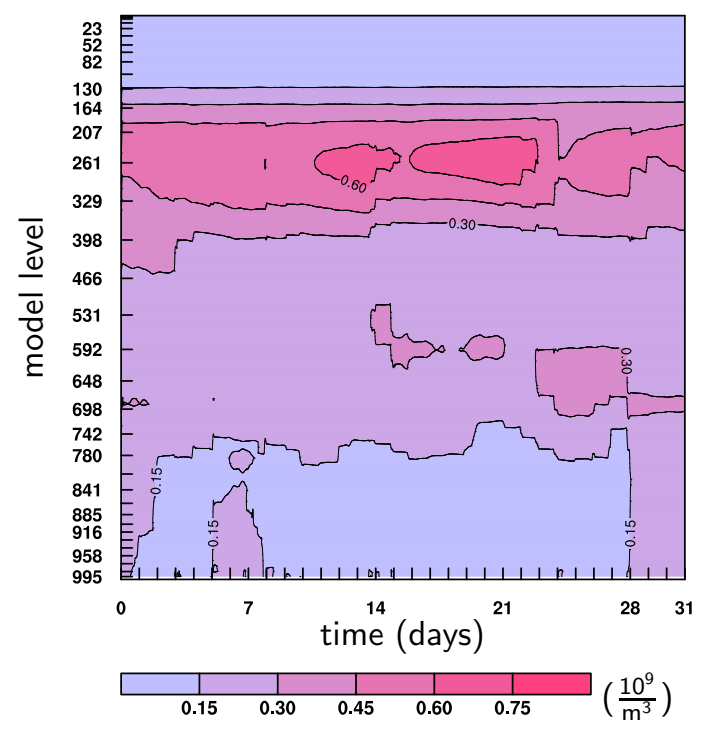

(b)

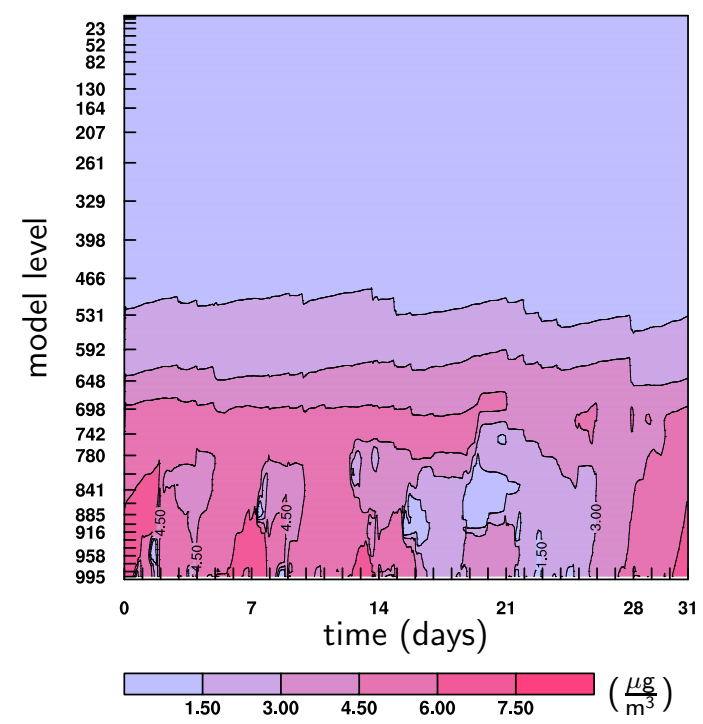

Fig. 7. Total aerosol number (a) and mass (b) concentrations in simulation for the ARM aerosol IOP, 1-31 May 2003. The model levels in the figure roughly correspond to pressure levels.

The diurnally varying photochemical production of $\mathrm{H}_{2} \mathrm{SO}_{4}$ is calculated at each time step for climatologically representative concentrations of the hydroxyl radical $(\mathrm{OH})$ and sulphur dioxide $\left(\mathrm{SO}_{2}\right)$ from previous simulations with AGCM4.

No feedbacks are included between aerosols and meteorological results in the simulations so that meteorological situations are identical in simulations for different numerical treatments of aerosol size distributions.

\subsection{Experiment setup}

The simulations were performed for the Southern Great Plains (SGP) site (at $36^{\circ} 37^{\prime} \mathrm{N} 97^{\circ} 30^{\prime} \mathrm{W}$ ) of the U.S. Department of Energy Atmospheric Radiation Measurement Program (ARM). The simulation period is from 1-31 May 2003, which overlaps with the ARM May 2003 Aerosol Intensive Operations (IOP) period (e.g. Feingold et al., 2006).

The model has been used to simulate the time evolution of the ammonium sulphate $\left[\left(\mathrm{NH}_{4}\right)_{2} \mathrm{SO}_{4}\right]$ aerosol size distribution at the SGP site for a particle radius range from 0.05 to $1.75 \mu \mathrm{m}$. Different numbers of sections have been used in different simulations to test the convergence of the numerical solutions for increasing numbers of section, similar to the comparisons in the previous sections.

For the present study, the winds were prescribed based on hourly large-scale analysis results. Temperature and humidity in SCM4 were forced using hourly analyzed advective tendencies for temperature and humidity. Results for temperature and humidity were additionally nudged towards analyzed temperature and humidity profiles. The analysis of meteorological results is based on numerical weather pre- diction products with additional constraints from surface and top-of-the-atmosphere measurements (Xie et al., 2004).

Analysis results were not available for initialization or forcing of chemical tracers in the present study. Horizontal transport processes were therefore neglected in the simulations. An iterative approach was employed in order to minimize the impact of the model spin-up period on the results. The model was repeatedly integrated for the same time period. Each simulation was initialized with concentrations from a previous model integration. This procedure was repeated for a total simulation period of 5 months until the aerosol concentrations were nearly in equilibrium. Only the results of the last month of the simulations were analyzed.

\subsection{Results}

Figure 7 shows the integrated $\left(\mathrm{NH}_{4}\right)_{2} \mathrm{SO}_{4}$ aerosol number and mass concentrations in the particle radius range from 0.05 to $1.75 \mu \mathrm{m}$ from a simulation with the PLA method with 40 sections for the aerosol number and mass concentrations $(i=1, \ldots, 40)$. In the simulation, the highest aerosol number concentrations occur in the upper troposphere. Favourable conditions, such as low temperatures and high relative humidities, lead to high nucleation rates at these levels (e.g. Seinfeld and Pandis, 1998). However, aerosol particles at these levels are relatively small so that very little mass is associated with these particles. The maximum in aerosol mass concentration occurs in the lower troposphere where concentrations of $\mathrm{SO}_{2}$ are high and condensation leads to efficient growth of the particles. 
(a)

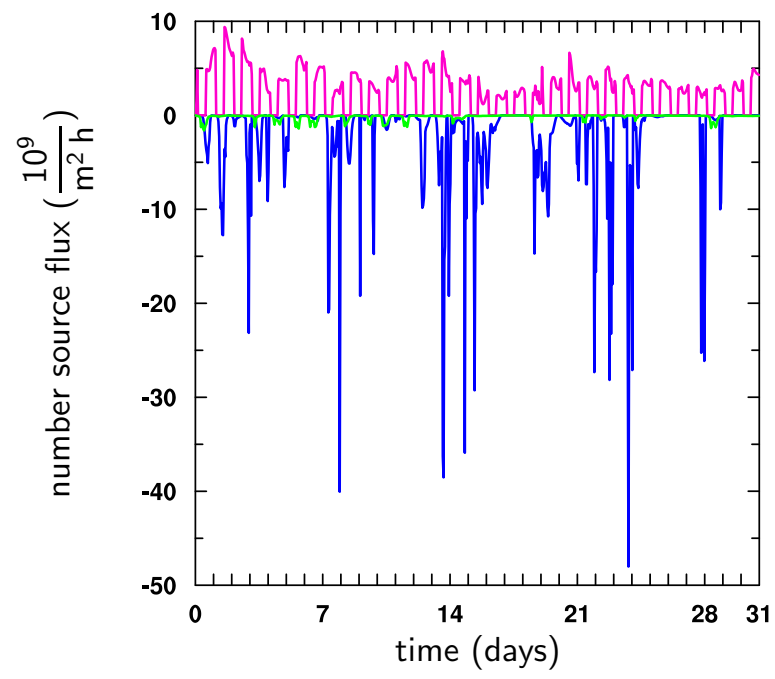

(b)

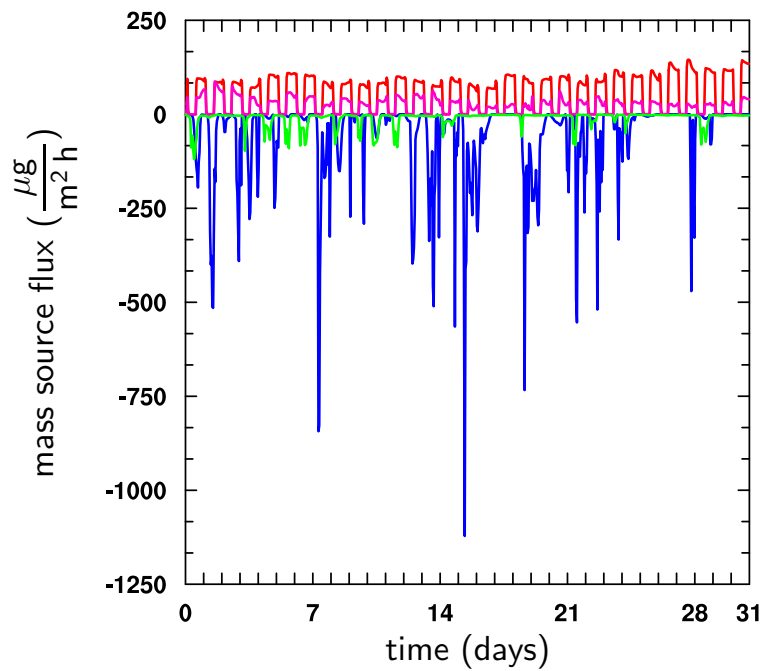

Fig. 8. Sources and sinks of column-integrated aerosol number (a) and mass (b) concentrations during the simulation. Sources are nucleation (pink lines), condensation (red line). Sinks are wet deposition (blue lines) and gravitational settling (green lines).

The simulated concentrations are quite variable in time. Most of the variability in the concentrations arises from intermittent wet deposition events. Simulated wet deposition rates are particularly high during the time period 14-24 May (Fig. 8). A large fraction of the precipitation results from deep convection in the simulation.

Variations in the magnitudes of the sources and sinks of aerosols are also connected to variations in the sizes of the aerosol particles (Fig. 9a). A single size mode is simulated for the aerosol mass, corresponding to the particle accumulation mode in the atmosphere. The deposition of aerosols does not directly lead to any noticeable changes in particle size. However, the model typically predicts increases in particle size during the time periods following large wet deposition events. This is presumably partly caused by a reduced total aerosol surface area. Consequently, the condensation of $\mathrm{H}_{2} \mathrm{SO}_{4}$ onto the aerosol particles that have not been removed by wet deposition is more efficient compared to the time period before the event. Additionally, the reduction in total surface area leads to increased nucleation rates during the time period following large wet deposition events (see Eq. A3) which eventually leads to the re-establishment of an accumulation mode that more or less resembles the mode that existed prior the wet deposition event.

There is also some evidence for increased production of small particles after wet deposition events from the results for the aerosol number size distribution in Fig. 10a. An additional mode occurs between about $D_{p}=0.1$ and $0.2 \mu \mathrm{m}$ in the simulations during these time periods.

As already mentioned, the primary purpose of the simulations is to test the performance of the PLA method and to compare results from this method with results from the modified TFL and SMB approaches. Results in Figs. 9 and 10 serve as an illustration of the approach in this study.

The most accurate simulations of the aerosol processes were obtained from control simulations with a total of 80 aerosol tracers for the PLA (e.g. Fig. 9a), the modified TFL (e.g. Fig. 9c), and SMB (e.g. Fig. 9e) methods, corresponding to 40 sections for the PLA and modified TFL methods and 80 sections for the SMB approach. Results of the control simulations agree very well with each other in terms of concentrations and sizes of the particles and their timing.

However, results of the comparison in Sects. 3 and 4 indicate that the accuracy of the SMB approach is systematically lower than the accuracy of the PLA method, even at these relatively large number of sections (e.g. Fig. 4). This explains why results using the SMB approach are characterized by slightly weaker maxima of the aerosol mass size distribution compared to the results of the PLA and modified TFL methods. Also note that, in contrast to the other results, results for the aerosol number size distribution for the SMB approach in Fig. 10e are diagnostic results (as previously described).

Results displayed in Figs. 9 and 10a, c, and e give evidence that the solutions from the different methods have converged to very nearly the same solution. It therefore appears to be appropriate to select results from one of the different methods at a large number of sections and to use these results as a reference in comparisons with simulations at low number of sections. This allows quantitative comparisons of the performance of each method for different number of sections, similar to the comparisons to an exact solution in Sects. 3 and 4. However, in contrast to previous sections, this approach 
(a)

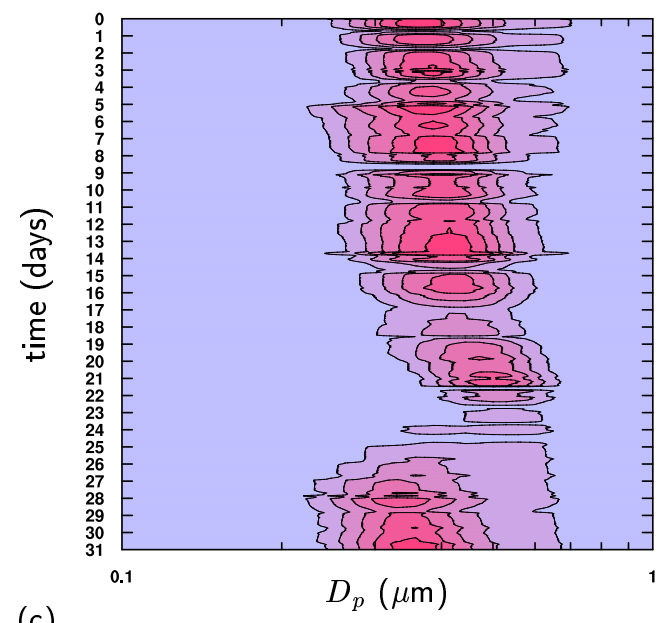

(c)

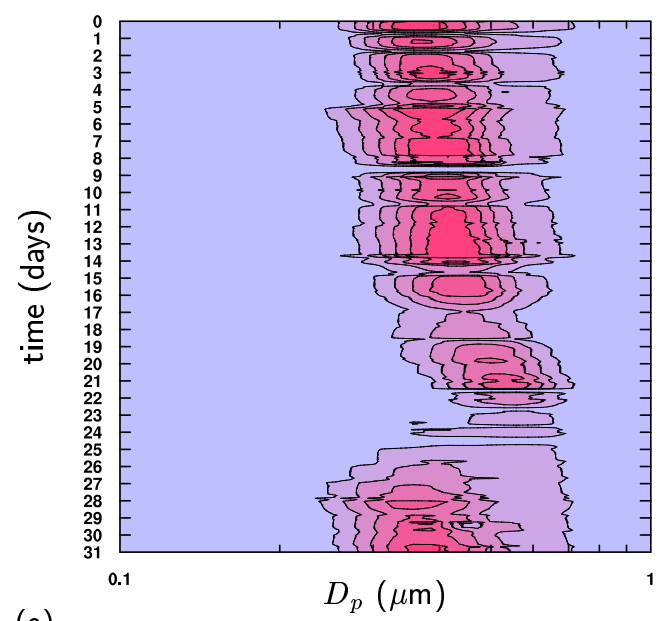

(e)

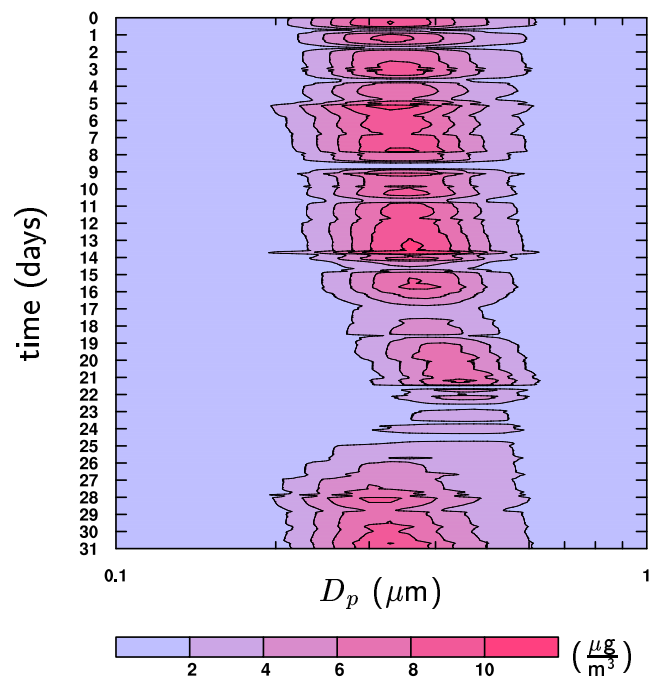

(b)

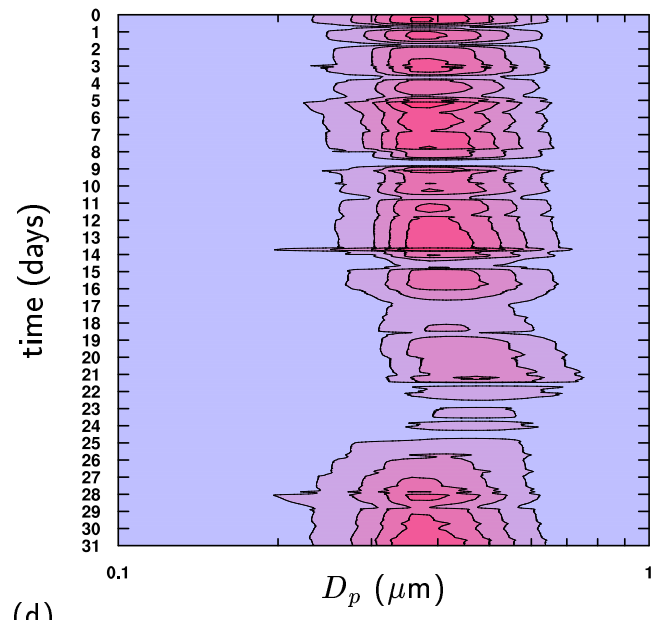

(d)

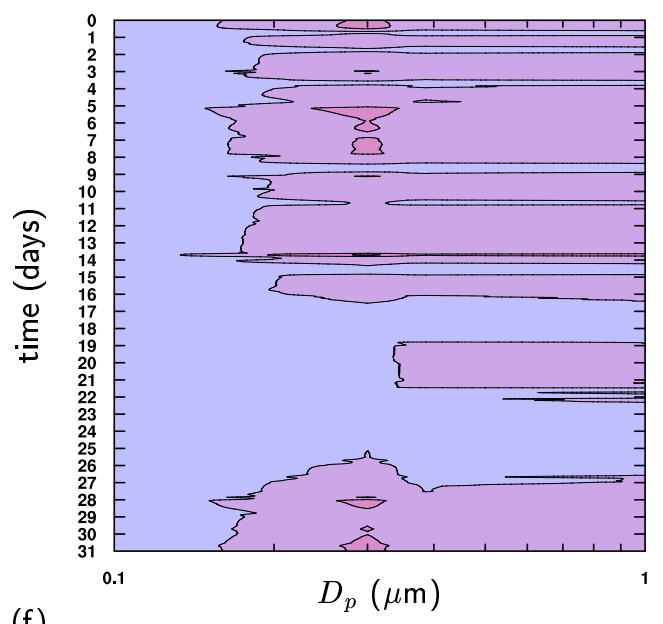

(f)

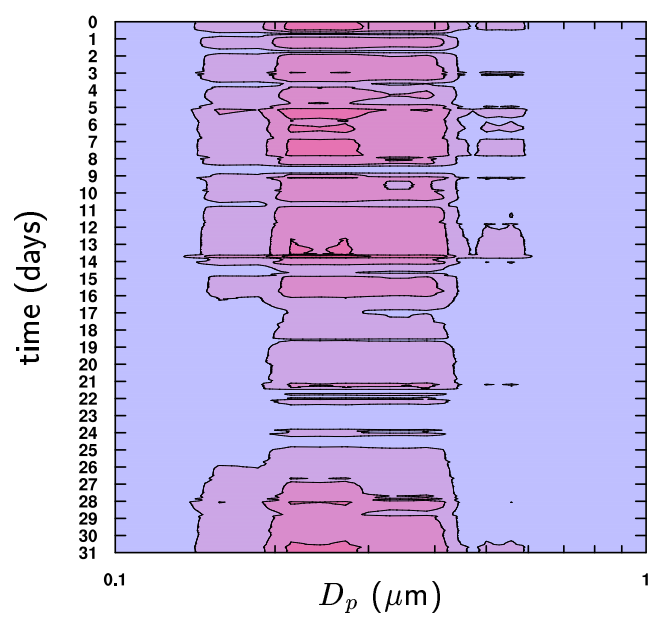

Fig. 9. Aerosol mass size distributions in the first model layer near the surface. (a) and (b) refer to results from simulations with the PLA method, (c) and (d) to simulations with the modified TFL approach, (e) and (f) to simulations with the SMB approach. Results for simulations with a large number of sections are displayed in the left column (40 sections for (a) and (c) and 80 sections for (e)) and results for a low number of sections in the right column ( 3 sections for $\mathrm{b}$ and $\mathrm{d}$ and 10 sections for $\mathrm{f}$ ). Results in a serve as a reference (see text). 
(a)

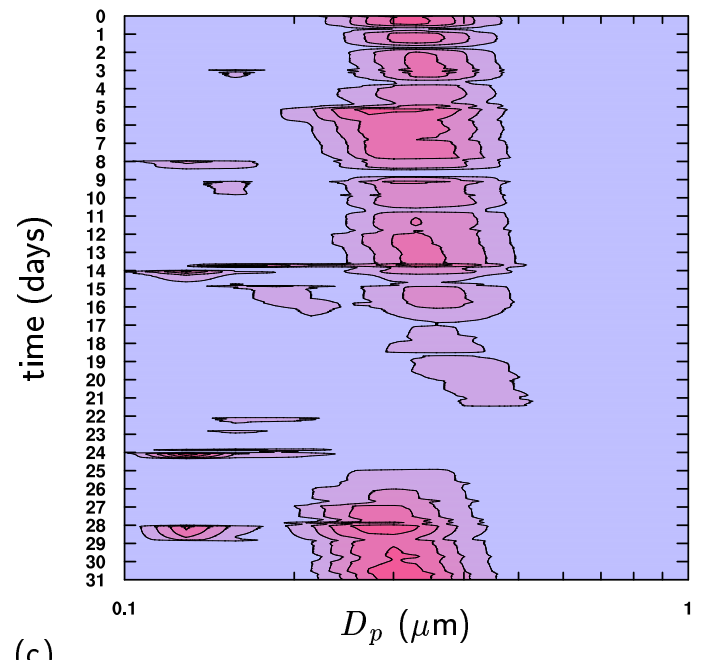

(c)

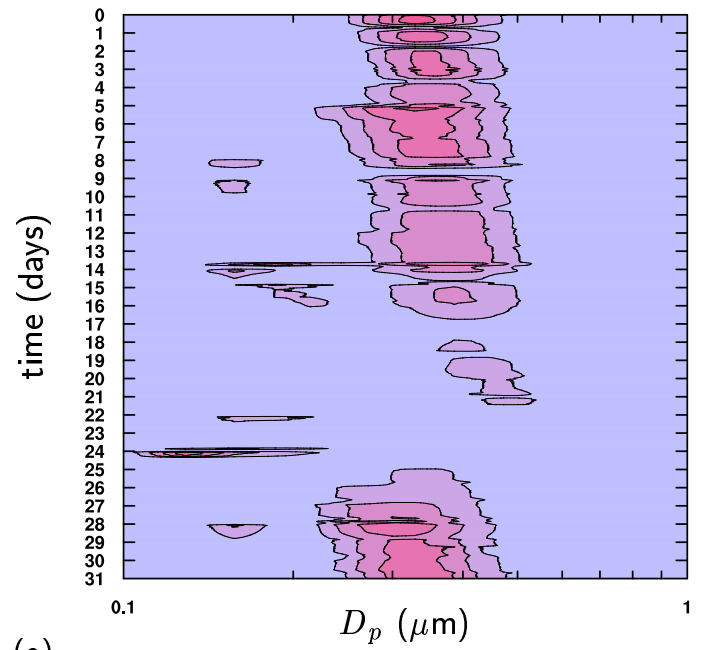

(e)
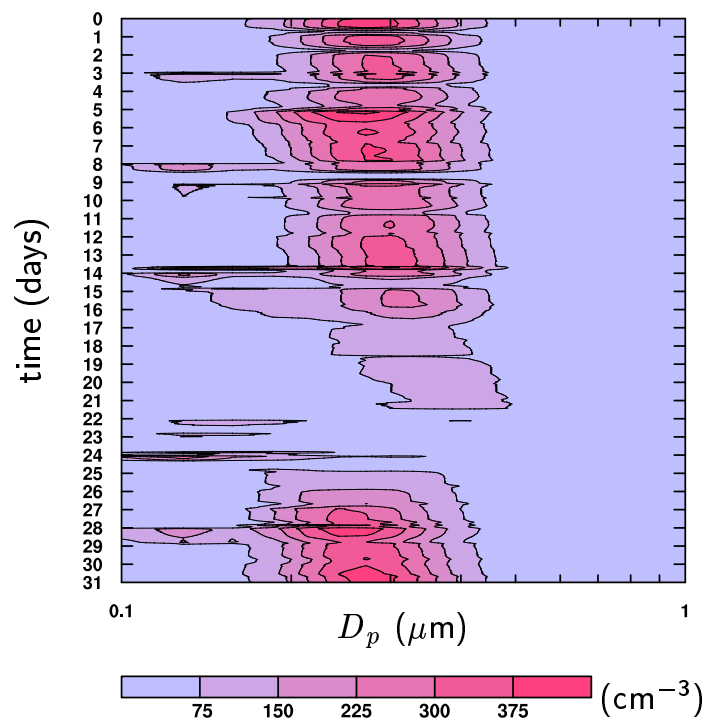

(b)

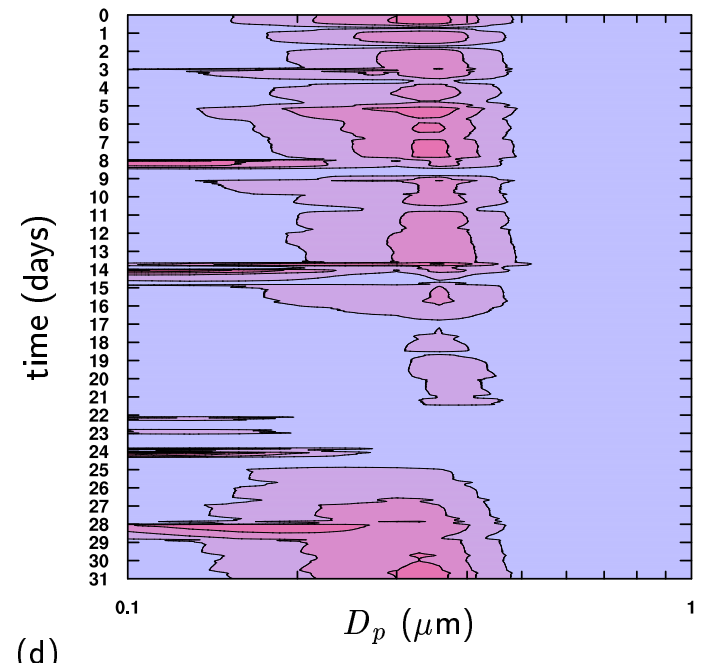

(d)

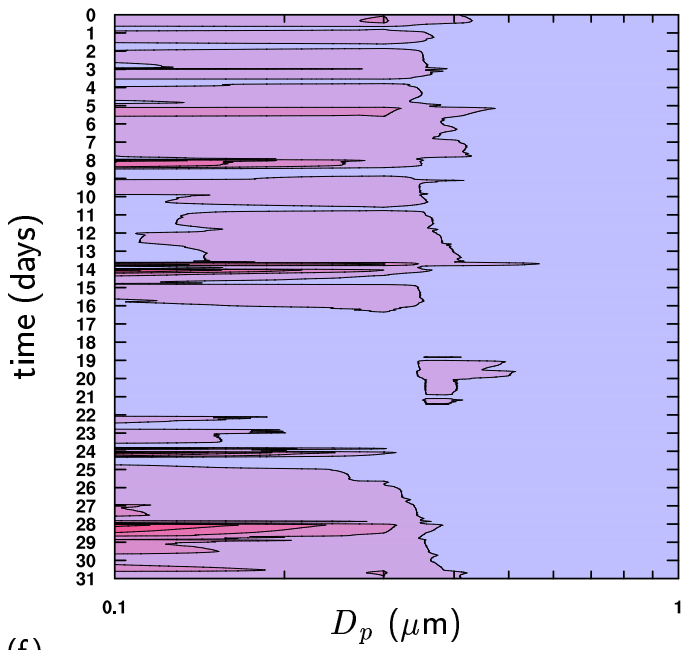

(f)

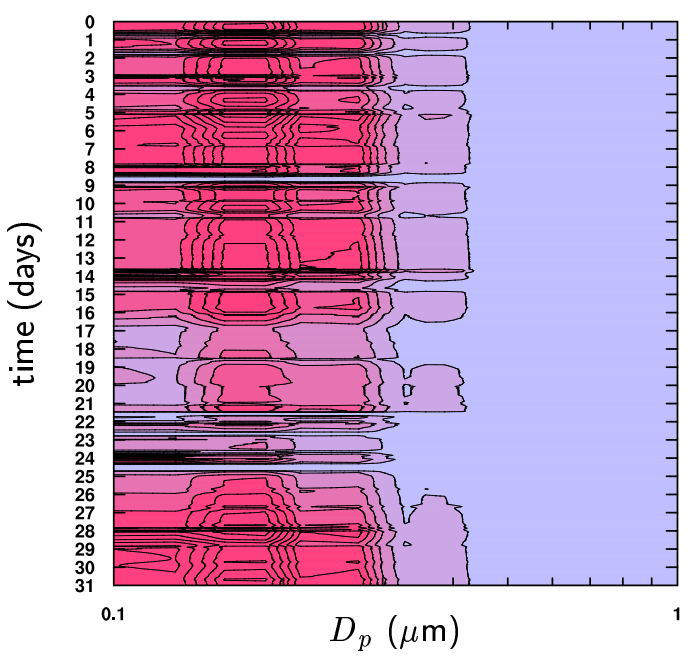

Fig. 10. Similar to Fig. 9, for the aerosol number size distribution. 
circumvents the use of an exact solution as a reference. In the following, results from the simulation with the PLA method in Figs. 9a and 10a are used as a reference.

The accuracy of the PLA method depends only weakly on the number of sections in the simulation. Results for $3 \mathrm{sec}-$ tions (i.e. 6 tracers) with the PLA method are still quite similar to the reference (Figs. 9b and 10b). The main differences are slightly weaker maxima and broader size spectra.

In contrast, there is a marked reduction in the quality of the results of the modified TFL and SMB approach for a reduced number of sections. A simulation with 6 aerosol tracers (modified TFL approach) respectively 10 aerosol tracers (SMB approach) produces much broader size distributions and therefore reveals very little about the temporal evolution in particle size as apparent in results of the reference experiment (Figs. 9d and $\mathrm{f}$ compared to Fig. 9a). The number size distributions are shifted towards much smaller particle sizes compared to the reference simulation. The two distinct particle size modes that are apparent in Figs. 10a, c, and e now appear as one single mode.

Artifacts such as those simulated for the SMB approach are typical of the upstream method, which has been used for the discretization of the condensation process for this approach (see Appendix A3). This method is only first-order accurate and thus tends to produce strong numerical diffusion (e.g. Bott, 1989). The introduction of the polynomial approximation approach (Sect. 3) should lead to improvements regarding the treatment of particle growth. However, as already shown in Sect. 3, this method would only be useful for relatively well resolved size distributions and would not lead to conservation of aerosol number in the simulation.

Results of the SMB approach for 10 sections (Figs. 9f and 10f) have an appreciably lower accuracy than the results of the PLA method for 3 sections (Figs. $9 \mathrm{~b}$ and 10b). This gives evidence that the application of the SMB method in interactive simulations of aerosol dynamical processes leads to larger errors compared to what might be expect from the results in Sect. 3. Comparisons in that section showed that PLA and SMB methods performed about equally well at these numbers of sections.

In analogy to the discussion in Sects. 3 and 4, Fig. 11 shows rms differences for simulations with the PLA, modified TFL, and SMB methods for different simulations with different number of sections (i.e. 1, 3, 10, 40, and $80 \mathrm{sec}-$ tions). Similar to Figs. 9 and 10, the accuracy of the results generally increases with increasing number of sections for all methods. However, the increase is less monotonic than in Fig. 4 because of a lower number of cases and the fact that section-integrated number and mass concentrations are not prescribed for the current results.

The PLA method produces a systematically higher accuracy than the SMB method. The same is true in comparison to the modified TFL approach, except for results for $10 \mathrm{sec}-$ tions, which give similar rms differences.
The difference in accuracy between the double-moment methods (i.e. PLA and modified TFL) and the SMB method is more apparent for the aerosol number concentration than it is for the aerosol mass concentration owing to the fact that the aerosol number concentration is a purely diagnostic quantity in the context of the SMB approach (Eq. A7) whereas it is predicted in the simulations with the double-moment methods.

Similar to the conclusion in Sect. 3, the accuracy of the results with the PLA method is only weakly sensitive to the choice of $\psi_{m, i}$ (Fig. 11).

\subsection{Comparison with observations}

It is interesting to compare the results of the simulations in Fig. 9 to observations at the SGP site during the ARM Aerosol IOP. An optical particle counter (OPC, Particle Measuring Systems Model PCASP-X) provided size distribution data in 31 size classes for particle diameters between 0.1 and $10 \mu \mathrm{m}$ (Sheridan et al., 2001). The aerosol sample stream that enters the OPC is heated to a relative humidity of $40 \%$ (or less) so that the effect of aerosol water is negligible. This allows a direct comparison between the observed and simulated size distributions. For the current study, the observed aerosol number concentrations in each size class were converted into an aerosol mass size distribution based on the assumption of spherical particles with a density of $1.769 \mathrm{~kg} \mathrm{~m}^{-3}$.

Observations are available for limited time periods during the IOP. Hourly average results for these time periods are shown in Fig. 12. Similar to the simulations (Fig. 9), a single particle mode has been observed in the range from 0.1 to $1 \mu \mathrm{m}$. Also, the maxima in the aerosol size distribution occur at similar particle sizes. However, the observed size distributions are considerably broader and more variable. It is likely that at least part of the differences are caused by the omission of horizontal transport of aerosols. Other likely causes are the omission of aerosol in-cloud production, coagulation, contributions of non-sulphate aerosols, as well the simplified treatments of wet deposition and photochemical production of $\mathrm{H}_{2} \mathrm{SO}_{4}$ in the simulations. Although the parameterized physics is incomplete, the results in Fig. 11 give evidence that the PLA method provides a much more accurate and efficient platform for aerosol modelling than the SMB method.

\section{Conclusions}

Aerosol physical and chemical processes generally depend on aerosol number, mass, and other moments of the aerosol size distribution. Currently, various models predict aerosol mass based on numerical approaches that do not guarantee continuity of aerosol number or other moments. A variety of multi-moment schemes has also been proposed for use in atmospheric models. However, there is no apparent 
(a)

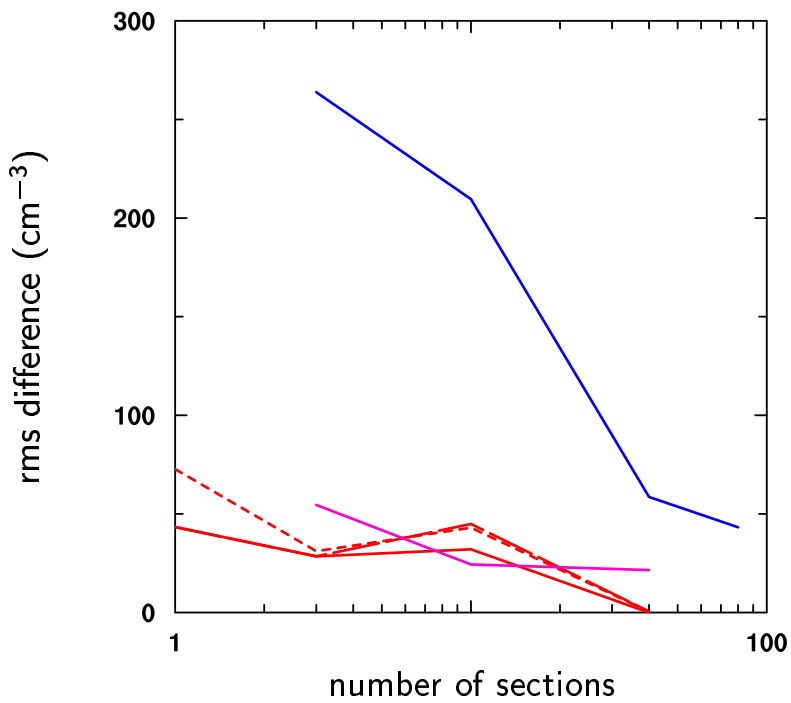

(b)

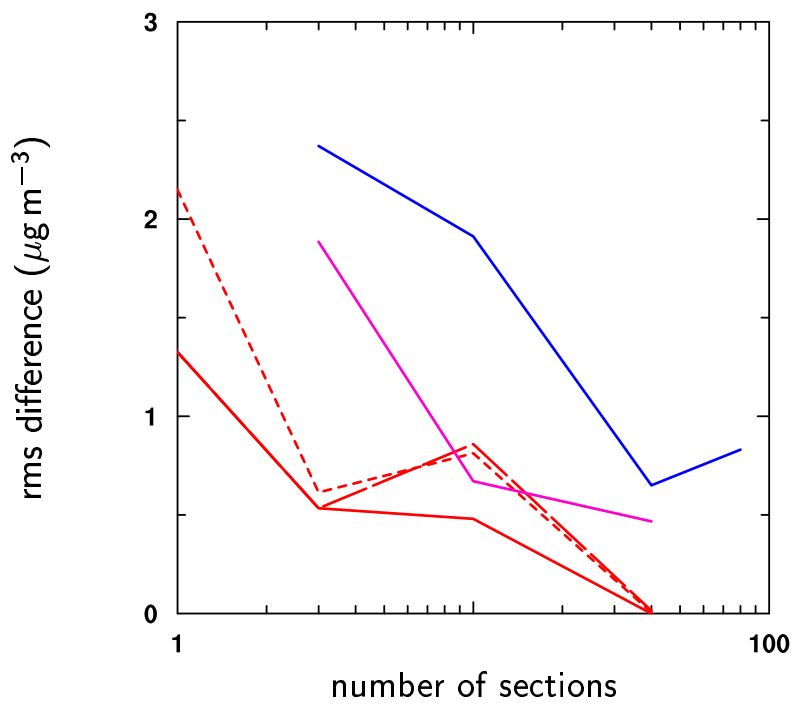

Fig. 11. Mean rms differences for simulated size distributions for aerosol number (a) and mass (b) in the first model layer. The differences are relative to the results of the simulation with the PLA method for 40 sections. Dark blue lines refer to the SMB approach, pink lines to the modified TFL approach, and red lines to the PLA method for optimal choice of $\psi_{m, i}$ (full lines), $\psi_{m, i}=1$ (long dashed lines), and $\psi_{m, i}=4$ (short dashed lines).

convergence in methodology for these schemes. Additionally, considerable computational costs are typically incurred from treatments of aerosol dynamical processes in models.

The piecewise log-normal approximation method (PLA) that is proposed here addresses these concerns by combining a sectional representation of the size distribution with an analytical description of the size distribution. The basic idea is that a sectional treatment is appropriate for large particle size scales (i.e. $\delta \varphi>\Delta \varphi_{\star}$ ). However, unresolved features of the size distribution on smaller scales (i.e. $\delta \varphi<\Delta \varphi_{\star}$ ) may be well approximated in terms of analytical basis functions within each section. The advantage of log-normal basis functions is that a wide range of conditions can be described and that it is straightforward to obtain any desired statistical moment of the distribution. The latter is particularly important for applications of the PLA method to the development of parameterizations.

A computationally efficient method is proposed to compute two of the free parameters of the log-normal basis functions from the number and mass concentrations in each section. The remaining third parameter is taken to be constant. Although it should in principle be possible to optimally specify this parameter, this study gives evidence for a relatively weak dependency of the results of the method on the value of this parameter.

An application of the PLA method to the approximation of observed size distributions gives evidence for high accuracy of the method for a wide range of the number of sections. In this example, results of the PLA method are at least

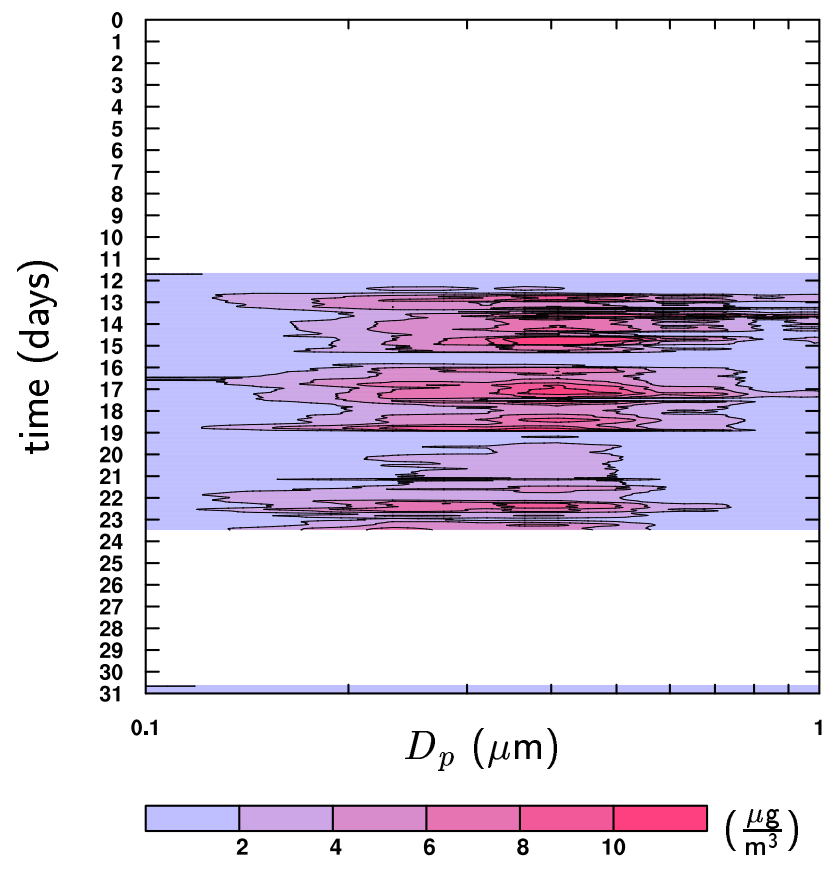

Fig. 12. Observed aerosol mass size distribution at the SGP site during the ARM aerosol IOP.

as accurate as the results of the traditional single-moment bin approach for a three times larger number of sections in the single-moment bin approach. The PLA method also produces systematically better agreement with the observations 
than double-moment bin schemes based on the work by Tzivion et al. (1987) or approximations in terms of polynomials.

The superior performance of the PLA method compared to the single- and double-moment bin methods is even more apparent from applications of the methods to prognostic simulations with a single column model. The processes included in these simulations are vertical transport, nucleation, condensation, gravitational settling, and wet deposition for ammonium sulphate aerosol.

Although the PLA method performs very well in applications to observed size distributions, the condensation equation, and single column model simulations in this study, it clearly is not possible to prove the efficiency of the method based on these tests alone. It will be necessary to study the performance of the method in additional applications to activation and chemical modifications in clouds, coagulation, etc. for more general conclusions. These tests will be done in the future. Despite the limited number of applications of the PLA method in the current study, the results appear to be useful tests of the method owing to the importance of the studied processes in the atmosphere and the difficulties to efficiently model these processes based on commonly considered single- and double-moment methods.

Results from additional tests of the method and implementations in the CCCma Atmospheric General Circulation Model will be reported in subsequent publications.

\section{Appendix A}

\section{Parameterization of nucleation and condensation}

The growth of aerosol particles due to condensation of sulphuric acid $\left(\mathrm{H}_{2} \mathrm{SO}_{4}\right)$ and ammonia $\left(\mathrm{NH}_{3}\right)$ can be expressed using Fick's law (e.g. Seinfeld and Pandis, 1998). $\mathrm{NH}_{3}$ is typically highly abundant in the troposphere (e.g. Dentener and Crutzen, 1994). Hence, it can be safely assumed that condensation of $\mathrm{NH}_{3}$ leads to nearly immediate and complete reaction of $\mathrm{H}_{2} \mathrm{SO}_{4}$ to $\left(\mathrm{NH}_{4}\right)_{2} \mathrm{SO}_{4}$ in the particles. Furthermore, the concentration of $\mathrm{H}_{2} \mathrm{SO}_{4}$ is approximately nil at the surface of the particles. This appears to be a good approximation owing to the very low volatility of $\mathrm{H}_{2} \mathrm{SO}_{4}$. Under these assumptions, the time evolution of the particle radius $R_{p d}$ for dry (i.e. solid) aerosol can be expressed by

$\frac{d R_{p d}}{d t}=\frac{c_{g} C}{R_{p d}}$

with the concentration $C$ of $\mathrm{H}_{2} \mathrm{SO}_{4}$ (in $\mathrm{kg} \mathrm{m}^{-3}$ ) and

$c_{g}=\left(\frac{M_{\left(\mathrm{NH}_{4}\right)_{2} \mathrm{SO}_{4}}}{M_{\mathrm{H}_{2} \mathrm{SO}_{4}}}\right) \frac{D F A e^{\Delta \varphi_{w}}}{\rho_{p d}}$.

$M_{\left(\mathrm{NH}_{4}\right)_{2} \mathrm{SO}_{4}}$ and $M_{\mathrm{H}_{2} \mathrm{SO}_{4}}$ refer to the molecular weights of $\left(\mathrm{NH}_{4}\right)_{2} \mathrm{SO}_{4}$ and $\mathrm{H}_{2} \mathrm{SO}_{4}$ (in kg mol$\left.{ }^{-1}\right)$. $\rho_{p d}$ denotes the density of the dry aerosol (in $\mathrm{kg} \mathrm{m}^{-3}$ ). $D$ represents the diffusivity of $\mathrm{H}_{2} \mathrm{SO}_{4}$ in air (in $\mathrm{m}^{2} \mathrm{~s}^{-1}$ ). $F$ and $A$ are dimensionless and size-dependent factors which account for non-continuum effects and imperfect surface accommodation (von Salzen et al., 2000). Note that unity is used for the mass accommodation coefficient in $A$ in this study. This value appears to be in qualitatively good agreement with results of recent theoretical and laboratory studies (Kulmala and Wagner, 2001). Finally, $\Delta \varphi_{w}$ refers to the difference between wet and dry particle size owing to the aerosol water content, i.e.

$\Delta \varphi_{w}=\ln \left(\frac{R_{p w}}{R_{p d}}\right)$,

for dry and wet particle radii, $R_{p d}$ and $R_{p w}$. In this study, $\Delta \varphi_{w}$ has been determined from thermodynamic equilibrium for mean concentrations in each section (Gong et al., 2003).

The time evolution of the $\mathrm{H}_{2} \mathrm{SO}_{4}$ concentration due to gasphase production, nucleation, and condensation can be described by:

$$
\begin{aligned}
& \frac{\mathrm{d} C}{\mathrm{~d} t}=P-k C^{s} \\
& -4 \pi C D \sum_{i} \int_{\varphi_{i-1 / 2}}^{\varphi_{i+1 / 2}} F A R_{p w} n(\varphi) \mathrm{d} \varphi .
\end{aligned}
$$

In Eq. (A3), $P$ represents the production of $\mathrm{H}_{2} \mathrm{SO}_{4}$ from oxidation of sulphur dioxide by the hydroxyl radical (Stockwell and Calvert, 1983). The following term represents the losses from binary homogeneous nucleation for $\mathrm{H}_{2} \mathrm{SO}_{4}$ and water vapour, with temperature and humidity dependent parameters $k$ and $s$ (e.g. von Salzen et al., 2000).

The last term in Eq. (A3) represents the condensation of $\mathrm{H}_{2} \mathrm{SO}_{4}$. It follows from Eq. (A1) from integration over the size distribution. $R_{p w}$ is calculated from $\Delta \varphi_{w}$ and the dry particle size $R_{p d}=R_{0} \exp (\varphi)$ (Eq. A2). Note that the number size distribution in the integral in Eq. (A3) is a function of the dry particle size with constant section boundaries $\varphi_{i \pm 1 / 2}$. This approach is sometimes used in aerosol models to increase the numerical accuracy of simulations with varying aerosol water contents. With this approach, the concentrations of the total aerosol (including water) can be easily diagnosed based on the results for the size distribution for dry particles and Eq. (A2).

Sensitivity tests give evidence for rapid adjustment of $C$ toward equilibrium for a wide range of atmospheric conditions that exist in large-scale atmospheric models. On the other hand, changes of $P, k, s, D, F, A, \Delta \varphi_{w}$, and $n(\varphi)$ are typically much smaller compared to changes in $C$ over a model time step ( $\Delta t \approx 30 \mathrm{~min}$ ). Hence, Eq. (A3) is integrated under the assumption of a constant aerosol size distribution during the time step.

\section{A1 Application of PLA method}

In order to simplify the numerical approach for the PLA method, the size-dependence of the term $F A$ in Eqs. (A3) 
and (A1) is approximated by

$F A \approx(F A)_{i-1 / 2}$

$+\frac{(F A)_{i+1 / 2}-(F A)_{i-1 / 2}}{e^{\varphi_{i+1 / 2}}-e^{\varphi_{i-1 / 2}}}\left(e^{\varphi}-e^{\varphi_{i-1 / 2}}\right)$

for $\varphi_{i-1 / 2} \leq \varphi \leq \varphi_{i+1 / 2}$.

Equation (A1) is used to obtain a linearized expression for the change in dry particle radius during a single model time step, i.e.

$R_{p d}\left(t_{0}+\Delta t\right)=R_{p d}\left(t_{0}\right)+\frac{c_{g}\left(t_{0}\right)}{R_{p d}\left(t_{0}\right)} \int_{t_{0}}^{t_{0}+\Delta t} C d t$,

where the integral results from integration of Eq. (A3) as described in the previous section. This procedure ensures that the change in particle mass from condensation of $\mathrm{H}_{2} \mathrm{SO}_{4}$ matches the change in $\mathrm{H}_{2} \mathrm{SO}_{4}$ concentration from integration of Eq. (A3).

Calculations for each section according to Eq. (A5) are followed by a remapping procedure so that the section boundaries for dry particles remain unchanged at each time step in the model. The remapping procedure is a straightforward integration over the size distribution for the particle radii after condensation (Eq. A5), based on Eqs. (4) and (5).

For the treatment of nucleation it is assumed that the size distribution of the freshly produced particles is a deltafunction for a particle size corresponding to the size of the smallest particles in the first section $i=1$. The amount of those particles is determined from integration of the nucleation term in Eq. (A3) over $\Delta t$.

\section{A2 Application of modified TFL approach}

In analogy to Eq. (14), size distributions can be defined for any required moment of the distribution, i.e.

$$
\begin{aligned}
\left(\frac{\mathrm{d} \mu_{k}}{\mathrm{~d} \varphi}\right)_{\mathrm{mTFL}, i} & =R_{p, i-1 / 2}^{k} a_{m, i}\left(\frac{\varphi_{i+1 / 2}-\varphi}{\Delta \varphi_{\star}}\right) \\
& +R_{p, i+1 / 2}^{k} b_{m, i}\left(\frac{\varphi-\varphi_{i-1 / 2}}{\Delta \varphi_{\star}}\right),
\end{aligned}
$$

for the same coefficients $a_{m, i}$ and $b_{m, i}$ as in Eq. (14).

Equation (A6) is used to solve Eq. (A3). Similar to the PLA method, Eq. (A4) is used to account for the sizedependence of the term $F A$ in Eq. (A3).

The approximated number and mass size distributions according to Eqs. (13) and (14), violate the fundamental relationship

$$
\frac{\mathrm{d} M}{\mathrm{~d} \varphi}=m_{p} \frac{\mathrm{d} N}{\mathrm{~d} \varphi}
$$

for $\varphi_{i-1 / 2}<\varphi<\varphi_{i+1 / 2}$. However, the approximated number and mass size distributions satisfy this relationship for particle sizes $\varphi=\varphi_{i-1 / 2}$ and $\varphi_{i+1 / 2}$ for each section (although the results may differ for adjacent sections). It is therefore desirable to apply Eq. (A5) to particles with sizes corresponding to the boundaries of the sections. This leads to new number and mass size distributions for these particles after condensation. By using the simplifying assumption that particle growth does not cause the size distributions to become nonlinear, these results can then be linearly interpolated to give new size distributions for all other particles. Similar to the procedure outlined in Sect. A1, remapping of the size distributions onto the original sections and integration yields new aerosol number and mass concentrations for each aerosol tracer.

\section{A3 Application of SMB approach}

For the application of the single-moment bin approach, it is assumed that the number size distribution within each section can be approximated by

$n(\varphi) \approx \frac{N_{i}}{\Delta \varphi_{\star}}$,

for $\varphi_{i-1 / 2} \leq \varphi \leq \varphi_{i+1 / 2}$, with

$N_{i} \approx \frac{M_{i}}{\frac{4 \pi}{3} \rho_{p d} R_{0}^{3} \exp \left(3 \varphi_{c, i}\right)}$,

and

$\varphi_{c, i}=\varphi_{i-1 / 2}+\frac{1}{2} \Delta \varphi_{\star}$.

It is further assumed that

$F A R_{p w} \approx\left(F A R_{p w}\right)_{i-1 / 2}$
$+\frac{\left(F A R_{p w}\right)_{i+1 / 2}-\left(F A R_{p w}\right)_{i-1 / 2}}{e^{\varphi_{i+1 / 2}}-e^{\varphi_{i-1 / 2}}}\left(e^{\varphi}-e^{\varphi_{i-1 / 2}}\right)$

for $\varphi_{i-1 / 2} \leq \varphi \leq \varphi_{i+1 / 2}$.

Similar to the application of the PLA method, Eq. (A3) is integrated to obtain the change $\Delta C$ in $\mathrm{H}_{2} \mathrm{SO}_{4}$ concentration over $\Delta t$. Let $\Delta C_{\mathrm{cnd}}$ be the contribution of condensation (i.e. from last term in Eq. A3) to $\Delta C$. Mass continuity then implies that the aerosol mass changes according to

$\Delta M_{i}=-\left(\frac{M_{\left(\mathrm{NH}_{4}\right)_{2} \mathrm{SO}_{4}}}{M_{\mathrm{H}_{2} \mathrm{SO}_{4}}}\right) \Delta C_{\mathrm{cnd}}$.

for particles with $\varphi_{i-1 / 2} \leq \varphi \leq \varphi_{i+1 / 2}$ at the beginning of the time step. A change in aerosol mass from condensation is accompanied by a change in particle size $\Delta \varphi_{c, i} . \Delta \varphi_{c, i}$ can be obtained from Eq. (A7) under the assumption that $N_{i}=$ const.:

$\Delta \varphi_{c, i}=\frac{1}{3} \ln \left(1+\frac{\Delta M_{i}}{M_{i}}\right)$.

On the other hand, section boundaries are not allowed to change between time steps, similar to the application of the PLA method. This requires a redistribution of mass between 
different sections. Hence, the mass concentration at time $t=t_{0}+\Delta t$ is calculated from

$\boldsymbol{M}\left(t_{0}+\Delta t\right)=\mathbf{T}\left[\boldsymbol{M}\left(t_{0}\right)+\Delta \boldsymbol{M}\right]$,

where $\boldsymbol{M}$ and $\Delta \boldsymbol{M}$ are vectors with elements $M_{i}$, respectively $\Delta M_{i}$. T is a matrix with elements

$$
\begin{aligned}
t_{i j} & =H\left(\Delta \varphi_{(i-1 / 2) j}^{-}\right) H\left(\Delta \varphi_{(i-1 / 2) j}^{+}\right) \frac{\Delta \varphi_{(i-1 / 2) j}^{+}}{\Delta \varphi_{\star}} \\
& +H\left(\Delta \varphi_{(i+1 / 2) j}^{-}\right) H\left(\Delta \varphi_{(i+1 / 2) j}^{+}\right) \frac{\Delta \varphi_{(i+1 / 2) j}^{-}}{\Delta \varphi_{\star}},
\end{aligned}
$$

with

$$
\begin{aligned}
& \Delta \varphi_{(i \pm 1 / 2) j}^{-}=\varphi_{t,(i \pm 1 / 2) j}-\varphi_{j-1 / 2}, \\
& \Delta \varphi_{(i \pm 1 / 2) j}^{+}=\varphi_{j+1 / 2}-\varphi_{t,(i \pm 1 / 2) j},
\end{aligned}
$$

and

$\varphi_{t,(i \pm 1 / 2) j}=\varphi_{i \pm 1 / 2}-\Delta \varphi_{c, j}$.

Aerosol mass resulting from nucleation is inserted into the first section according the change in $\mathrm{H}_{2} \mathrm{SO}_{4}$ concentration from nucleation over $\Delta t$ (Eq. A3).

\section{Appendix B}

\section{Parameterization of gravitational settling}

The terminal settling velocity of a spherical aerosol particle is

$v_{t}=\frac{2}{9} \frac{R_{p w}^{2} \rho_{p w} g C_{c}}{\mu}$,

with the gravitational acceleration $g$, density of the wet aerosol $\rho_{p w}$, and slip correction factor

$C_{c}=1+\frac{\lambda}{R_{p w}}\left[1.257+0.4 \exp \left(-\frac{1.1 R_{p w}}{\lambda}\right)\right]$,

and mean free path of air $\lambda$ (Seinfeld and Pandis, 1998).

In order to simplify the discretization and increase the efficiency of the numerical solution, the approximation

$\exp \left(-\frac{1.1 R_{p w}}{\lambda}\right) \approx \exp \left(-\frac{1.1 R_{0} e^{\varphi_{c, i}+\Delta \varphi_{w}}}{\lambda}\right)$

is used for $\varphi_{i-1 / 2} \leq \varphi \leq \varphi_{i+1 / 2}$ in Eq. (B2), with $\varphi_{c, i}$ from Eq. (A8).

\section{B1 Application of PLA method}

Equation (B1) is used to calculate effective terminal settling velocities for particle number $\left(v_{t n, i}\right)$ and mass $\left(v_{t m, i}\right)$ in each section $i$ from integration over the section, i.e.

$v_{t n, i}=\frac{1}{N_{i}} \int_{\varphi_{w, i-1 / 2}}^{\varphi_{w, i+1 / 2}} v_{t} n\left(\varphi-\Delta \varphi_{w}\right) \mathrm{d} \varphi$,

$v_{t m, i}=\frac{1}{M_{i}} \frac{4 \pi}{3} \rho_{p, i} R_{0}^{3} \int_{\varphi_{w, i-1 / 2}}^{\varphi_{w, i+1 / 2}} v_{t} e^{3 \varphi} n\left(\varphi-\Delta \varphi_{w}\right) \mathrm{d} \varphi$,

with $\varphi_{w, i \pm 1 / 2}=\varphi_{i \pm 1 / 2}+\Delta \varphi_{w}$.
B2 Application of modified TFL approach

Equations (B3) and (B4) are also used for the TFL approach for linear $R_{p}^{k} n\left(\varphi-\Delta \varphi_{w}\right)$ according to Eqs. (13), (14), and (A6).

\section{B3 Application of SMB approach}

Effective terminal settling velocities are obtained for $M_{i}$ from Eq. (B1) for $R_{p w}=R_{0} \exp \left(\varphi_{c, i}+\Delta \varphi_{w}\right)$.

Acknowledgements. I would like to thank S. Ghan and two other anonymous reviewers for their very helpful comments. The author further thanks S. Xie for providing the single column model forcing data and G. Feingold, N. McFarlane, W. Merryfield, and J. Scinocca for discussions. This research was partially supported by the Climate Change Action Fund (CCAF) and Environment Canada.

Edited by: G. Feingold

\section{References}

Ackermann, I. J., Hass, H., Memmesheimer, M., Ebel, A., Binkowski, F. S., and Shankar, U.: Modal aerosol dynamics model for Europe: Development and first applications, Atmos. Environ., 32, 2981-2999, 1998.

Binkowski, F. S. and Shankar, U.: The regional particulate matter model 1. Model description and preliminary results, J. Geophys. Res., 100, 21 191-21 209, 1995.

Birmili, W., Wiedensohler, A., Heintzenberg, J., and Lehmann, K.: Atmospheric particle number size distribution in central Europe: Statistical relations to air masses and meteorology, J. Geophys. Res., 106, 32 005-32 018, 2001.

Bott, A.: A positive definite advection scheme obtained by nonlinear renormalization of the advective fluxes, Mon. Wea. Rev., 117, 1006-1015, 1989.

Bott, A.: A flux method for the numerical solution of the stochastic collection equation, J. Atmos. Sci., 55, 2284-2293, 1998.

Dentener, F. and Crutzen, P. J.: A 3-dimensional model of the global ammonia cycle, J. Atmos. Chem., 19, 331-369, 1994.

Dhaniyala, S. and Wexler, A. S.: Numerical schemes to model condensation and evaporation of aerosols, Atmos. Environ., 30, 919928, 1996.

Feingold, G.: Modeling of the first indirect effect: Analysis of measurement requirements, Geophys. Res. Lett., 30, 1997, doi:10.1029/2003GL017967, 2003.

Feingold, G., Furrer, R., Pilewski, P., Remer, L. A., Min, Q., and Jonsson, H.: Aerosol indirect effect studies at Southern Great Plains during the May 2003 intensive operations period, J. Geophys. Res., 111, D05514, doi:10.1029/2004JD005648, 2006.

Feller, W.: An introduction to probability theory and its applications, Whiley, New York, 1971.

Gelbard, F.: Modeling multicomponent aerosol particle growth by vapor condensation, Aerosol Sci. Technol., 12, 399-412, 1990.

Gelbard, F. and Seinfeld, J. H.: Simulation of multicomponent aerosol dynamics, J. Colloid Interface Sci., 78, 485-501, 1980.

Gilliani, N. V., Schwartz, S. E., Leaitch, W. R., Strapp, J. W., and Isaac, G. A.: Field observations in continental stratiform clouds: 
Partitioning of cloud particles between droplets and unactivated interstitial aerosols, J. Geophys. Res., 100, 18 687-18 706, 1995.

Gong, S. L., Barrie, L. A., Blanchet, J.-P., von Salzen, K., Lohmann, U., Lesins, G., Spacek, L., Zhang, L. M., Girard, E., Lin, H., Leaitch, R., Leighton, H., Chylek, P., and Huang, P.: Canadian Aerosol Module: A size-segregated simulation of atmospheric aerosol processes for climate and air quality models - 1. Module development, J. Geophys. Res., 108, 4007, doi:10.1029/2001JD002002, 2003.

Harrington, D. Y. and Kreidenweis, S. M.: Simulations of sulfate aerosol dynamics - Part II. Model intercomparison, Atmos. Environ., 32, 1701-1709, 1998.

Jacobson, M. Z.: Development and application of a new air pollution modeling system - II. Aerosol module structure and design, Atmos. Environ., 31, 131-144, 1997.

Kerminen, V.-M. and Wexler, A. S.: Growth laws for atmospheric aerosol particles: An examination of the bimodality of the accumulation mode, Atmos. Environ., 29, 3263-3275, 1995.

Kleinman, L. I., Daum, P. H., Imre, D. G., Lee, J. H., Lee, Y.N., Nunnermacker, L. J., Springston, S. R., Weinstein-Lloyd, J., and Newman, L.: Ozone production in the New York city urban plume, J. Geophys. Res., 105, 14 495-14 511, 2000.

Korhonen, H., Lehtinen, K. E. J., Pirjola, L., Napari, I., and Vehkamäki, H.: Simulation of atmospheric nucleation mode: A comparison of nucleation models and size distribution representations, J. Geophys. Res., 108, 4471, doi:10.1029/2002JD003305, 2003.

Kulmala, M. and Wagner, P. E.: Mass accommodation and uptake coefficients - a quantitative comparison, J. Aerosol Sci., 32, 833841, 2001.

Laprise, R. and Girard, E.: A spectral general circulation model using a piecewise-constant finite element representation on a hybrid vertical coordinate system, J. Climate, 3, 32-52, 1990.

Lohmann, U., von Salzen, N., McFarlane, K., Leighton, H. G., and Feichter, J.: Tropospheric sulphur cycle in the Canadian general circulation model, J. Geophys. Res., 104, 26 833-26 858, 1999.

Lurmann, F. W., Wexler, A. S., Pandis, S. N., Musarra, S., Kumar, N., and Seinfeld, J. H.: Modelling urban and regional aerosols - II. Application to California's south coast air basin, Atmos. Environ., 31, 2695-2715, 1997.

McFarlane, N. A., Scinocca, J. F., Lazare, M., Harvey, R., Verseghy, D., and Li, J.: The CCCma third generation atmospheric general circulation model (AGCM3), Internal report, Canadian Centre for Climate Modelling and Analysis, pp. 23, 2006.

McGraw, R.: Description of aerosol dynamics by the quadrature method of moments, Aerosol Sci. Technol., 27, 255-265, 1997.

Meng, Z. Y., Dabdub, D., and Seinfeld, J. H.: Size-resolved and chemically resolved model of atmospheric aerosol dynamics, J. Geophys. Res., 103, 3419-3435, 1998.

Pilinis, C., Capaldo, K. P., Nenes, A., and Pandis, S. N.: MADM - A new multicomponent aerosol dynamics model, Aerosol Sci. Technol., 32, 482-502, 2000.

Remer, L. A., Kaufman, Y. J., and Holben, B. N.: Interannual variation of ambient aerosol characteristics on the east coast of the United States, J. Geophys. Res., 104, 2223-2231, 1999.

Russell, L. M. and Seinfeld, J. H.: Size- and composition-resolved externally mixed aerosol model, Aerosol Sci. Technol., 28, 403416, 1998.

Seigneur, C.: A model of sulfate aerosol dynamics in atmospheric plumes, Atmos. Environ., 16, 2207-2228, 1982.

Seigneur, C., Hudischewskyi, A. B., Seinfeld, J. H., Whitby, K. T., Whitby, E. R., Brock, J. R., and Barnes, H. M.: Simulation of aerosol dynamics: A comparative review of mathematical models, Aerosol Sci. Technol., 5, 205-222, 1986.

Seinfeld, J. H. and Pandis, S. N.: Atmospheric chemistry and physics: From air pollution to climate change, Whiley, New York, 1998.

Sheridan, P. J., Delene, D. J., and Ogren, J. A.: Four years of continuous surface aerosol measurements from the Department of Energy's Atmospheric Radiation Measurement Program Southern Great Plains Cloud and Radiation Testbed site, J. Geophys. Res., 106, 20 735-20 747, 2001.

Stockwell, W. R. and Calvert, J. G.: The mechanism of HO-SO reaction, Atmos. Environ., 17, 2231-2235, 1983.

Tzivion, S., Feingold, G., and Levin, Z.: An efficient numerical solution to the stochastic collection equation, J. Atmos. Sci., 44, 3139-3149, 1987.

von Salzen, K. and Schlünzen, K. H.: A prognostic physicochemical model of secondary and marine inorganic multicomponent aerosols I. Model description, Atmos. Environ., 33, 767576, 1999a.

von Salzen, K. and Schlünzen, K. H.: A prognostic physicochemical model of secondary and marine inorganic multicomponent aerosols II. Model tests, Atmos. Environ., 33, 1543-1552, $1999 b$.

von Salzen, K., Leighton, H. G., Ariya, P. A., Barrie, L. A., Gong, S. L., Blanchet, J.-P., Spacek, L., Lohmann, U., and Kleinman, L. I.: Sensitivity of sulphate aerosol size distributions and CCN concentrations over North America to $\mathrm{SO}_{\mathrm{x}}$ emissions and $\mathrm{H}_{2} \mathrm{O}_{2}$ concentrations, J. Geophys. Res., 105, 9741-9765, 2000.

von Salzen, K., McFarlane, N. A., and Lazare, M.: The role of shallow convection in the water and energy cycles of the atmosphere, Climate Dyn., 25, 671-688, doi:10.1007/s00382-00551-2, 2005

Warren, D. R. and Seinfeld, J. H.: Simulation of aerosol size distribution evolution in systems with simultaneous nucleation, condensation, and coagulation, Aerosol Sci. Technol., 4, 31-43, 1985.

Whitby, E., Stratmann, F., and Wilck, M.: Merging and remapping modes in modal aerosol dynamics models: A "Dynamic Mode Manager", J. Aerosol Sci., 33, 623-645, 2002.

Whitby, E. R.: Modal aerosol dynamics modeling, Aerosol Sci Technol., 27, 673-688, 1997.

Whitby, K. T.: The physical characteristics of sulfur aerosols, Atmos. Environ., 12, 135-159, 1978.

Whitby, K. T.: Determination of aerosol growth rates in the atmosphere using lumped aerosol dynamics, J. Aerosol Sci., 12, 174178, 1981.

Wilck, M. and Stratmann, F.: A 2-D multicomponent modal aerosol model and its application to laminar flow reactors, J. Aerosol Sci., 28, 959-972, 1997.

Wilson, J., Cuvelier, C., and Raes, F.: A modeling study of global mixed aerosol fields, J. Geophys. Res., 106, 34 081-34 108, 2001.

Wright, D. L., Kasibhatla, P. S., McGraw, R., and Schwartz, S. E.: Description and evaluation of a six-moment aerosol microphysical module for use in atmospheric chemical transport models, J. Geophys. Res., 106, 20 275-20 291, 2001. 
Xie, S., Cederwall, R. T., and Zhang, M.: Developing long-term single-column model/cloud system-resolving model forcing data using numerical weather prediction products constrained by surface and top of the atmosphere observations, J. Geophys. Res., 109, D01104, doi:10.1029/2003JD004045, 2004.

Yu, S., Kasibhatla, P. S., Wright, D. L., Schwartz, S. E., and McGraw, R.: Moment-based simulation of microphysical properties of sulfate aerosols in the eastern United States: Model description, evaluation, and regional analysis, J. Geophys. Res., 108, 4353, doi:10.1029/2002JD002890, 2003.

Zender, C. S., Bian, H., and Newman, D.: Mineral dust entrainment and deposition (DEAD) model: Description and 1990s dust climatology, J. Geophys. Res., 108, 4416, doi:10.1029/2002JD002775, 2003.
Zhang, Y., Seigneur, C., Seinfeld, J. H., Jacobson, M. Z., and Binkowski, F. S.: Simulation of aerosol dynamics: A comparative review of algorithms used in air quality models, Aerosol Sci. Technol., 31, 487-514, 1999.

Zhang, Y., Easter, R. C., Ghan, S. J., and Abdul-Razzak, H.: Impact of aerosol size representations on modeling aerosol-cloud interactions, J. Geophys. Res., 107, 4558, doi:10.1029/2001JD001549, 2002. 